\title{
Lithium depletion and angular momentum transport in F-type and G-type stars in Galactic open clusters
}

\author{
T. Dumont ${ }^{1,2}$, C. Charbonnel ${ }^{2,3}$, A. Palacios ${ }^{1}$, and S. Borisov ${ }^{2,4}$ \\ ${ }^{1}$ LUPM, Université de Montpellier, CNRS, Place Eugène Bataillon, 34095 Montpellier, France \\ 2 Department of Astronomy, University of Geneva, Chemin Pegasi 51, 1290 Versoix, Switzerland \\ e-mail: thibaut.dumont@unige.ch \\ 3 IRAP, CNRS UMR 5277 \& Université de Toulouse, 14 avenue Edouard Belin, 31400 Toulouse, France \\ 4 Sternberg Astronomical Institute, M.V. Lomonosov Moscow State University, Universitetsky prospect 13, Moscow 119234, Russia \\ Received 15 April 2021 / Accepted 14 July 2021
}

\begin{abstract}
Context. Open clusters provide unambiguous clues to understand the evolution of ${ }^{7} \mathrm{Li}$ at the surface of low-mass stars and its possible correlation with stellar rotation, which is a challenge for both stellar hydrodynamics and Galactic chemical evolution.

Aims. We aim to quantify the efficiency of the transport processes for both angular momentum and chemicals that are required to explain simultaneously the observed behaviour of surface ${ }^{7} \mathrm{Li}$ (and ${ }^{9} \mathrm{Be}$ ) and rotation as well as the internal rotation profiles inferred from helio- and asteroseismology in F- and G-type main sequence stars.

Methods. We apply the model for the transport of angular momentum and chemicals that we tailored in a previous work for solartype stars to an extended range of initial masses and metallicities corresponding to F- an G-type stars in a sample of 20 Galactic open clusters. We evaluate its ability to explain the ${ }^{7} \mathrm{Li},{ }^{9} \mathrm{Be}$, and rotation periods observations. This model includes atomic diffusion, rotation-induced processes (for which we tested different prescriptions for shear turbulence), penetrative convection with a rotational dependence, parametric viscosity and turbulence, and magnetic braking.

Results. Over the entire range of masses, metallicities, and ages explored, we reproduce the evolution of the surface rotation rates and predict, for the first time, the observed anti-correlation between the surface rotation rate and ${ }^{7} \mathrm{Li}$ depletion as a consequence of the penetrative convection prescription. The ${ }^{7} \mathrm{Li}$ behaviour and its evolution with time is well reproduced for G-type stars. However, the ability of the model to reproduce the so-called ${ }^{7} \mathrm{Li}$ dip centred around $\sim 6600 \mathrm{~K}$ strongly depends on the adopted prescriptions for shear turbulence. It also requires a stellar mass dependence for the parametric viscosity adopted for the transport of angular momentum, similar to the behaviour predicted for the generation and luminosity of internal gravity waves generated by stellar convective envelopes. Finally, the model predicts internal rotation profiles in good agreement with asteroseismic constraints in main sequence stars.

Conclusions. We provide an efficient way to model G-type stars of different ages and metallicities successfully. However, the ${ }^{7} \mathrm{Li}$ and ${ }^{9} \mathrm{Be}$ dip constraints urgently call for further hydrodynamical studies to better model turbulence in stars, and for the exploration of physical processes such as tachocline mixing for the transport of chemicals and internal gravity waves for the transport of angular momentum. Finally, additional data for the internal rotation and for ${ }^{9} \mathrm{Be}$ in main sequence low-mass stars are definitively needed.
\end{abstract}

Key words. stars: abundances - stars: rotation - stars: evolution - stars: low-mass - open clusters and associations: general

\section{Introduction}

Understanding the evolution of ${ }^{7} \mathrm{Li}$ (hereafter $\mathrm{Li}$ ) in low-mass stars is one of the main challenges for stellar and Galactic astrophysics. Despite Li being a very scarce element, it is a tracer of Galactic chemical evolution (e.g., Spite \& Spite 1982; Matteucci et al. 1995; Romano et al. 2001; Travaglio et al. 2001; Prantzos 2012) and of transport processes that occur in stellar interiors (e.g., Charbonneau \& Michaud 1990; Montalban \& Schatzman 1996; Montalbán \& Schatzman 2000; Charbonnel \& Talon 2005; Talon \& Charbonnel 2010; Castro et al. 2016; Dumont et al. 2021, hereafter Paper I, and references therein). An overall picture of the different possibly involved processes is described in the reviews by Mathis (2013) and Aerts et al. (2019).

Observations of open clusters have provided numerous $\mathrm{Li}$ abundance data for stars of different ages over a large range of masses and metallicities (e.g., Boesgaard 1976, 1991; Duncan \& Jones 1983; Cayrel et al. 1984; Balachandran et al. 1988, 2011; Soderblom et al. 1990, 1993a; Thorburn et al.
1993; Garcia Lopez et al. 1994; Swenson et al. 1994; Jones et al. 1999; Boesgaard et al. 2003a,b; Sestito \& Randich 2005; Anthony-Twarog et al. 2009, 2018; Cummings et al. 2012, 2017; Deliyannis et al. 2019; Randich et al. 2020). It has been clearly evidenced that Li depletion increases with time and is linked to stellar mass (e.g., Deliyannis et al. 2000, for a review). At a given age, the Li behaviour as a function of the stellar effective temperature $\left(T_{\text {eff }}\right)$ shows two specific patterns. On the one hand, photospheric Li abundances of G-type stars decrease with decreasing effective temperature (decreasing mass). On the other hand, a group of F-type stars with $T_{\text {eff }}$ centred around $6600 \mathrm{~K}$ fall into the so-called Li dip which appears in open clusters older than 200 Myrs (e.g., Wallerstein et al. 1965; Boesgaard \& Tripicco 1986; Hobbs \& Pilachowski 1986; Soderblom et al. 1993b; Balachandran 1995; Steinhauer \& Deliyannis 2004; Boesgaard et al. 2016).

Classical stellar evolution (accounting only for convection as a mixing process in stellar interiors) predicts noticeable $\mathrm{Li}$ depletion during the pre-main sequence (PMS) when the base of 
the convective envelope is deep enough to reach the Li-burning temperature, which happens only for less massive stars (i.e., below $\sim 0.9-1.0 M_{\odot}$ at solar metallicity; e.g., Bodenheimer 1965; Pinsonneault 1997). However, it does not predict any further surface Li variation until the first dredge-up occurs after the stars leave the main sequence (MS), in striking contrast with the observational evidence of the steepening along the main sequence of the $\mathrm{Li}-T_{\text {eff }}$ trend of the cool side of the $\mathrm{Li}$ dip and of the Li dip itself.

The key role of rotation was pointed out as the cool edge of the Li dip coinciding with the so-called Kraft rotation break, as observed for instance in the Hyades (Boesgaard 1987) and in NGC 752 (Hobbs \& Pilachowski 1986). The Kraft break (Kraft 1967) corresponds to the transition stellar mass $\left(\simeq 1.2 M_{\odot}\right.$ at solar metallicity) where important structural changes occur in main sequence stars. In particular, stars more massive than the transition value have an extremely thin convective envelope, implying weaker or ineffective magnetic braking compared to cooler, less massive stars with a thick convective envelope that can sustain efficient stellar wind magnetic braking (e.g., Schatzman 1962; Weber \& Davis 1967; Matt et al. 2015; Kawaler 1988; Cummings et al. 2017; Deliyannis et al. 2019). The cool side of the dip also corresponds to the transition region where the convective envelope can efficiently generate internal gravity waves that transport angular momentum (Talon \& Charbonnel 2003, 2004, 2005), and to an internal structure where the stellar core is radiative while hotter MS stars host a convective core.

Different non-standard mixing-processes (beyond convection) have been explored to explain the observed Li features in F-type and G-type stars. This includes the following: convective overshooting or penetrative convection (Schlattl \& Weiss 1999; Brun et al. 2017; Baraffe et al. 2017; Jørgensen \& Weiss 2018, Paper I), atomic diffusion (Michaud 1986; Richer \& Michaud 1993; Turcotte et al. 1998), mass loss (Guzik \& Mussack 2010), planet accretion or migration (Montalbán \& Rebolo 2002; Castro et al. 2009), tachocline mixing (Brun et al. 1999, Paper I), internal gravity waves (Montalban 1994), rotation-induced mixing (Pinsonneault et al. 1990; Charbonnel et al. 1992; Palacios et al. 2003; Eggenberger et al. 2012a; Somers \& Pinsonneault 2016; Amard et al. 2016; Zhang et al. 2019), magnetic processes and instabilities (Charbonneau \& MacGregor 1993; Ruediger \& Kitchatinov 1996; Eggenberger et al. 2005, 2010), as well as different combinations of some of the above-mentioned processes where the transports of chemicals and angular momentum are intimately coupled (Richard et al. 1996; Charbonnel \& Talon 2005; Talon \& Charbonnel 2005; Deal et al. 2020; Semenova et al. 2020, Paper I).

Importantly, Li appears to be only one piece of a bigger puzzle that should also include the constraints from ${ }^{9} \mathrm{Be}$ (hereafter $\mathrm{Be}$ ) that burns at a slightly higher temperature than $\mathrm{Li}$ ( 3.5 MK and 2.5 MK, respectively; e.g. Pinsonneault 1997). Indeed, while the $\mathrm{Be}$ dip coincides with the $\mathrm{Li}$ dip (e.g., Deliyannis et al. 1998; Boesgaard et al. 2001, 2004a, 2020), $\mathrm{Be}$ is hardly depleted in the Sun, solar-like stars, and G-type stars (Balachandran \& Bell 1998; Boesgaard et al. 2003a,b, 2004b, 2016, 2020). The Be behaviour thus brings additional constraints to the possible origin of the observed behaviour of $\mathrm{Li}$ in F- and G-type stars and to the depth of the required mixing process. Last but not least, the difficulty is to find the actual connection between the transport of chemicals and the transport of angular momentum at play in stars of different spectral types along their evolution, to account simultaneously for the internal rotation profiles that can be deduced for the Sun and for some other stars thanks to asteroseismology (Kosovichev 1988; Elsworth et al. 1995; Mathur et al. 2008; Eff-Darwich et al. 2008; Marques et al. 2013; Benomar et al. 2015; Eggenberger et al. 2019a; García \& Ballot 2019).

In this work, we explore the chemical and rotational evolution of low-mass stars on the PMS and the MS for different stellar masses and metallicities that cover the range of Galactic open clusters with ages between 5 Myrs and 4 Gyrs. We study the effects and the relevance for these stars of different transport processes that we already explored and validated for the specific case of the solar-type stars (Paper I), and that depend on both mass and metallicity. In Sect. 2, we present the observational data that we used to constrain model predictions. In Sect. 3 we describe the input physics of the stellar models and the different processes implemented in the evolution code STAREVOL used for this work. In Sect. 4, we compare the observations for the Hyades and Praesepe open clusters with the predictions for $\mathrm{Li}, \mathrm{Be}$, and surface rotation of our non-standard model including meridional circulation, shear-induced turbulence, atomic diffusion, overshoot, and parametric viscosity and turbulence. In Sect. 5, we compare the model predictions with $\mathrm{Li}$ and rotational periods $\left(P_{\text {rot }}\right)$ data in a sample of open clusters over a broad range in age and metallicity. In Sect. 6, we discuss model predictions for the internal rotation and compare to asteroseismic measurements. We summarise our results and conclude in Sect. 7.

\section{Observational data}

We use observational data for a sample of Galactic open clusters. Their names, ages, metallicities, and distances to the Galactic centre are given in Table 1 along with bibliographical references from which the Li and Be surface abundances and rotation periods of individual stars were taken. We only take into account non-binary stars with confirmed membership as mentioned or flagged in the reference papers cited in Table 1.

\subsection{Lithium and Beryllium abundances}

In this work, we consider $\mathrm{Li}$ spectroscopic data for a sample of 14 open clusters (see Table 1 ) with $[\mathrm{Fe} / \mathrm{H}]$ values between -0.38 and +0.16 dex, and ages between 35 Myrs and 4 Gyrs: IC 2602, IC 2391, Pleiades, $\alpha$ Persei ( $\alpha$ Per), M 35, M 50, Coma Berenices (Coma Ber), Ursa Major (UMa), Hyades, NGC 6633, Praesepe, NGC 6819, NGC 2420, M 67, and NGC 2243. We adopted the meteoritic abundance $A(\mathrm{Li})=3.31^{1}$ (Asplund et al. 2009) as the original abundance of lithium.

All the original papers give 1D local thermodynamic equilibrium (LTE) Li abundances, except Jeffries et al. (2002), where they give non-local thermodynamic equilibrium (NLTE) $\mathrm{Li}$ abundances for NGC 6633 stars. As lithium abundances are known to be sensitive to non-LTE effects, we computed the $3 \mathrm{D}$ NLTE corrections $\left(\Delta_{\text {NLTE }}\right)$ to all the 1D LTE lithium abundances, using the code Breidablik ${ }^{2}$ by Wang et al. $(2021)^{3}$. To do so, for each star, we used the $[\mathrm{Fe} / \mathrm{H}], T_{\text {eff }}$, and $\log g$ values given in the original papers also providing the lithium

\footnotetext{
$1 A(X)=\log _{10}\left(N_{X} / N_{H}\right)+12$, where $N_{X}$ is the number density of element $X$.

2 https://github.com/ellawang44/Breidablik

3 In the case of the NGC 6633 stars from Jeffries et al. (2002), we first reversed their NLTE correction using the code of Carlsson et al. (1994) to obtain the 1D LTE abundances and then computed the same 3D NLTE corrections as for the other data.
} 
T. Dumont et al.: Lithium depletion and angular momentum transport in F-type and G-type stars in Galactic open clusters

Table 1. Main properties of the open clusters considered in this work, with the corresponding references for the Li and Be abundances and the rotation periods.

\begin{tabular}{|c|c|c|c|c|c|c|c|}
\hline Name & Age (Myrs) & Ref Age & {$[\mathrm{Fe} / \mathrm{H}]$} & Distance (kpc) & Ref Li & Ref Be & Ref $P_{\text {rot }}$ \\
\hline NGC 2362 & 5 & I & 0.00 & 9.11 & - & - & $\mathrm{a}$ \\
\hline NGC 2547 & 35 & I & $-0.14 \pm 0.10$ & 8.39 & - & - & $\mathrm{b}$ \\
\hline IC 2602 & 35 & II & $-0.02 \pm 0.02$ & 8.29 & 1 & - & - \\
\hline IC 2391 & 36 & II & $-0.03 \pm 0.02$ & 8.34 & 1 & & $\mathrm{c}$ \\
\hline Pleiades (Melotte 22) & 87 & II & $-0.01 \pm 0.05$ & 8.45 & 2 & - & $\mathrm{b} ; \mathrm{d}$ \\
\hline$\alpha \operatorname{Per}($ Melotte 20) & 87 & II & $+0.14 \pm 0.11$ & 8.48 & $3 ; 4$ & - & $\mathrm{c}$ \\
\hline M 35 (NGC 2168) & 150 & I & $-0.17 \pm 0.01$ & 9.24 & $5 ; 6$ & - & $\mathrm{e}$ \\
\hline M 50 (NGC 2323) & 150 & I & $0.00^{(\bullet)}$ & 9.10 & - & - & $b ; f$ \\
\hline NGC 2516 & 150 & I & $-0.06 \pm 0.05$ & 8.32 & - & - & $\mathrm{b}$ \\
\hline M 37 (NGC 2099) & 500 & I & $+0.02 \pm 0.05$ & 9.77 & - & - & $\mathrm{b} ; \mathrm{g}$ \\
\hline Coma Ber (Melotte 111) & 570 & III & $0.00 \pm 0.08$ & 8.35 & 7 & - & - \\
\hline UMa & 600 & IV & $-0.09^{(\star)}$ & $8.37^{(*)}$ & 7 & - & - \\
\hline Hyades (Melotte 25) & 720 & III & $+0.13 \pm 0.05$ & 8.38 & 8 & 9 & $\mathrm{~h} ; \mathrm{i}$ \\
\hline NGC 6633 & 770 & II & $-0.08 \pm 0.12$ & 8.00 & $10 ; 11$ & - & - \\
\hline Praesepe (NGC 2632) & 750 & II & $+0.16 \pm 0.08$ & 8.48 & 8 & 12 & $\mathrm{~b} ; \mathrm{j} ; \mathrm{k} ; \mathrm{l}$ \\
\hline NGC 6811 & 950 & I & $+0.03 \pm 0.01$ & 8.20 & - & - & $\mathrm{b} ; \mathrm{m}$ \\
\hline NGC 6819 & 2000 & II & $+0.09 \pm 0.01$ & 8.03 & 13 & - & $\mathrm{n}$ \\
\hline NGC 2420 & 2500 & $\mathrm{~V}$ & $-0.05 \pm 0.02$ & 10.68 & 14 & - & - \\
\hline M 67 (NGC 2682) & 3640 & II & $-0.01 \pm 0.04$ & 8.96 & 15 & - & - \\
\hline NGC 2243 & 4000 & VI & $-0.38 \pm 0.04$ & 10.58 & 16 & - & - \\
\hline
\end{tabular}

Notes. The $[\mathrm{Fe} / \mathrm{H}]$ values are from Netopil et al. (2016) and Gutiérrez Albarrán et al. (2020), except for UMa (Boesgaard et al. 2003a) and M 50 (Douglas et al. 2016). Distances to the Galactic centre are from Cantat-Gaudin et al. (2020), except for UMa (Ujjwal et al. 2020) and they are based on the Gaia DR2 measurements. ${ }^{(\bullet}[\mathrm{Fe} / \mathrm{H}]$ from Godoy-Rivera et al. $(2021) .{ }^{(\star)}[\mathrm{Fe} / \mathrm{H}]$ from Boesgaard et al. $(2003 \mathrm{a}) .{ }^{(*)}$ Distance to the Galactic centre from Ujjwal et al. (2020), assuming the Sun is at a distance of $8.34 \mathrm{kpc}$ from the Galactic centre (Cantat-Gaudin et al. 2020).

References. I: Godoy-Rivera et al. (2021); II: Bossini et al. (2019), III: Netopil et al. (2016); IV: Boesgaard et al. (2003a); V: Semenova et al. (2020); VI: Gutiérrez Albarrán et al. (2020). 1: Randich et al. (2001); 2:Bouvier et al. (2018); 3: Boesgaard et al. (2003b); 4: Balachandran et al. (2011); 5: Barrado y Navascués et al. (2001); 6: Jeffries et al. (2021); 7: Boesgaard et al. (2003a); 8: Cummings et al. (2017); 9: Boesgaard et al. (2016); 10: Jeffries (1997); 11: Jeffries et al. (2002); 12: Boesgaard et al. (2004b); 13: Deliyannis et al. (2019); 14: Semenova et al. (2020); 15: Pace et al. (2012); 16: François et al. (2013). a: Irwin et al. (2008); b: Godoy-Rivera et al. (2021); c: Irwin \& Bouvier (2009); d: Hartman et al. (2010); e: Meibom et al. (2009); f: Irwin et al. (2009); g: Hartman et al. (2008); h: Delorme et al. (2011); i: Douglas et al. (2016); j: Agüeros et al. (2011); k: Douglas et al. (2017); l: Douglas et al. (2019); m: Meibom et al. (2011); n: Meibom et al. (2015).

abundances (see Table 1). In the specific case of the Pleiades, Bouvier et al. (2018) do not give the surface gravity. We thus adopted $\log g=4.4$ for its stars, which appears to describe the typical surface gravity of $\mathrm{F}$ and $\mathrm{G}$ dwarfs ${ }^{4}$ well. The absolute values of NLTE corrections rarely exceed $0.1 \mathrm{dex}$; we consequently do not expect a significant impact on the results.

We also used the Be spectroscopic data as an additional constraint for the Hyades and Praesepe from Boesgaard et al. (2004b) and Boesgaard et al. (2016). Observations were directly extracted from Boesgaard et al. (2004b, 2016) without any modification. We adopted the meteoritic abundance $A\left({ }^{9} \mathrm{Be}\right)=1.41$ (Asplund et al. 2009) as the original abundance of beryllium.

\subsection{Age}

There is no self-consistent determination in the literature of the ages of all the clusters we consider here, and this task is out of the scope of this work. To be consistent with Paper I, we used the ages from Bossini et al. (2019, when available)

\footnotetext{
${ }^{4} \Delta_{\text {NLTE }}$ weakly depends on $\log g$ for most of the studied temperature range. For instance, for $T_{\text {eff }}=6000 \mathrm{~K}, A(\mathrm{Li})=2.5$ and $[\mathrm{Fe} / \mathrm{H}]=0$, $\Delta_{\mathrm{NLTE}}(\log g=4.4)-\Delta_{\mathrm{NLTE}}(\log g=4.0)=-0.0135$ dex, which is negligible. However, for $T_{\text {eff }} \approx 3900 \mathrm{~K}$ to $4500 \mathrm{~K}$, this difference is more significant and reaches values of about +0.08 dex. In our sample, only 17 out of the 92 stars of Pleiades are in this temperature range, therefore this should not have a significant impact on the result.
}

for the clusters with $\mathrm{Li}$ abundance measurements. For the clusters with rotation period measurements, we used the ages from Godoy-Rivera et al. (2021, when available). For the five clusters with $\mathrm{Li}$ data that were not studied in those two works, we took the ages quoted in the respective observation papers (see Table 2). Except for the Pleiades, the differences in age determinations from the literature weakly affect our conclusions (see discussion in Sect. 5.1).

\subsection{Effective temperature $T_{\text {eff }}$}

The effective temperatures used in this study were taken from the same original sources as the lithium (or beryllium) abundances for consistency (see Table 1). In rare cases, spectroscopic $T_{\text {eff }}$ are available (this is the case of M67 and NGC 2420). In most cases, $T_{\text {eff }}$ were determined using colour-temperature calibration relations from $(B-V),\left(V-I_{c}\right)$, or $(V-K)$ colour indices. For the 14 open clusters presented in Table 1, at least six different relations and methods were used. These relations differ by their metallicity dependence, the zero-point of their temperature scale, the colours used, etc. The relative consistency between the different calibration relations was tested in several previous studies (Huang et al. 2015; Casagrande et al. 2010; Meléndez et al. 2010). These studies emphasise that maximum differences of about $100 \mathrm{~K}$ can be found when applying the different temperature scales to dwarf stars in the metallicity and photometric domain considered here. We thus consider that the general 
Table 2. Transport processes considered in this work.

\begin{tabular}{lccc}
\hline \hline Process & Quantity & Adjusted parameter & Observational constraints \\
\hline Atomic diffusion & $\mathrm{C}$ & - & - \\
Meridional circulation & C \& AM & - & - \\
Shear-induced turbulence (R1 or R2) & C \& AM & - & Surface rotation of solar-type stars \\
Magnetic torque & AM & $K=7.5 \times 10^{30} \mathrm{erg}$ & Surface Li abundance of young solar-type stars \\
Penetrative convection & $\mathrm{C}$ & $d_{o v}=0.0325$ & Surface Li abundance of MS solar-type stars \\
Parametric turbulence & $\mathrm{C}$ & $\log T=6.42$ & Solar rotation profile \\
Vertical viscosity & $\mathrm{AM}$ & $v_{\mathrm{add}}=3.5 \times 10^{4} \mathrm{~cm}^{2} \mathrm{~s}^{-1}$ & \\
\hline
\end{tabular}

Notes. Transport processes (Col. 1) and values of the free parameters (when relevant) (Col. 3) with the corresponding observational constraints adjusted (Col. 4) for the best model for solar-type stars (Paper I) and adopted in this study. The flags in Col. 2 indicate the transported quantity (C for chemicals and AM for angular momentum).

agreement between the different temperature scales adopted in the original papers that we use in this work is satisfactory.

\subsection{Surface and internal rotation}

We compared the surface rotation predicted by our models with the observational data set gathered by Irwin \& Bouvier (2009), Gallet \& Bouvier (2015), and Godoy-Rivera et al. (2021) for low-mass stars among a sample of 12 open clusters with ages between 5 Myrs and 2 Gyrs: NGC 2362, NGC 2547, IC 2391, $\alpha$ Per, Pleiades, M 35, M 50, M 37, Hyades, Praesepe, NGC 6811, and NGC 6819 (see details in Table 1).

We constrained our model predictions for internal rotation using the asteroseismic measurements obtained by Benomar et al. (2015) for a sample of MS field stars observed by Kepler. We selected a sub-sample of four stars with metallicities close to that of the Hyades.

\section{Stellar evolution models - Input micro- and macro-physics}

\subsection{General assumptions}

We followed the conclusions of Paper I on different transport processes of chemicals and angular momentum (namely: rotation, penetrative convection, parametric turbulence, parametric viscosity, and atomic diffusion, as described below), which were tested with respect to $\mathrm{Li}$ abundances and surface and internal rotation constraints for solar-type stars. We used the nomenclature described in Paper I. For instance, model ${ }_{v}^{M} R 1_{A}^{T 6.42}$ is a model computed with an $\mathrm{R} 1$ prescription for rotation-induced turbulence and parametric viscosity $\left(v=v_{\text {add }}\right)$, penetrative convection $(A)$, parametric turbulence down to $\log T_{0}=6.42$, at median initial rotation velocity $(M)$, and with atomic diffusion. All the details about the corresponding prescriptions for the different processes are given below.

To a broader range of masses and metallicities, we applied the prescriptions that were identified to be the most relevant for solar-type stars. Models were computed in the mass range between $0.8 M_{\odot}$ and $1.5 M_{\odot}\left(\delta \mathrm{M}=0.1 M_{\odot}\right)$ for seven values of $[\mathrm{Fe} / \mathrm{H}](-0.4,-0.2,-0.1,-0.05,0,+0.10$, $+0.15)$ that cover the metallicity range of the Galactic open clusters described in Table 1. Computations started on the Hayashi track at the beginning of the deuterium burning phase on the PMS that we consider as the time zero of the evolution.

\subsection{Input physics}

We used the same updated version of the stellar evolution code STAREVOL as in Paper I (for general information and previous versions see Siess et al. 2000; Palacios et al. 2006; Decressin et al. 2009; Lagarde et al. 2012; Amard et al. 2019), which we refer to for details and references. The models presented in this work were computed with the same inputs physics (equation of state, opacities, nuclear reactions, model atmosphere, and mass loss). We used the same values for the mixing length parameter ( $\alpha_{\mathrm{MLT}}$, assuming the Schwarzschild criteria for convective stability) and the initial abundances that resulted from model calibrations on the Sun that were carried out for classical models (no transport of chemicals beyond convection), and for models including both rotation and atomic diffusion (respectively models C and R1 from Paper I).

\subsection{Atomic diffusion, penetrative convection, parametric turbulence}

Atomic diffusion was implemented according to Paquette et al. (1986) and Thoul et al. (1994). We did not take radiative accelerations into account. Their impact starts, however, to be non-negligible for stars with effective temperature higher than $\sim 6800 \mathrm{~K}$, which corresponds to $\sim 1.4 M_{\odot}$ at solar metallicity (Richer et al. 1998; Richard et al. 2002; Deal et al. 2018, 2020). This is discussed in Sect. 7.

Penetrative convection was treated as an overshoot and computed following the formalism of Augustson \& Mathis (2019) with the following expression for the diffusion coefficient:

$$
D_{A}(r) \approx D_{0}\left[1-\exp \left(-\exp \left(\frac{r-r_{b c z}}{d_{o v} \times\left(\frac{v}{v_{0}}\right)^{3 / 2}}+\frac{\mu}{\lambda}\right)\right)\right],
$$

where $D_{0}=\left(v_{\text {conv }} \times H_{\mathrm{p}} \times \alpha_{\mathrm{MLT}}\right) / 3$ is the convective turbulent diffusivity, with $v_{\text {conv }}$ being the mean velocity of the convective elements obtained from MLT, $\alpha_{\text {MLT }}$ being the mixing length parameter, and $H_{\mathrm{p}}$ being the pressure scale height; $r$ is the local radius; $r_{b c z}$ is the radius at the base of the convective zone; $\left(v / v_{0}\right)$ is the ratio of the velocity of the convective elements when taking rotation into account for the non-rotating inviscid value; and $d_{o v}=0.0325$ (as calibrated by Paper I to reproduce the Li abundance in solar-type stars in very young open clusters). The coefficients $\lambda=6 \times 10^{-3}$ and $\mu=5 \times 10^{-3}$ are as prescribed by Baraffe et al. (2017) based on the simulations of Pratt et al. (2017).

Parametric turbulence is defined according to Richer et al. (2000) and Richard et al. (2005) with the following prescription 
T. Dumont et al.: Lithium depletion and angular momentum transport in F-type and G-type stars in Galactic open clusters

Table 3. Models computed for the specific case of the Hyades and Praesepe open clusters at $[\mathrm{Fe} / \mathrm{H}]=+0.15$.

\begin{tabular}{lccc}
\hline \hline Model & $v_{\text {add }}\left(\mathrm{cm}^{2} \mathrm{~s}^{-1}\right)$ & $\mathrm{K}\left(10^{30} \mathrm{erg}\right)$ & Turbulence \\
\hline$C$ & 0 & 7.5 & none \\
${ }_{v} R 1_{A}^{T 6.42}$ & $3.5 \times 10^{4}$ & 7.5 & $D_{T 6.42}$ \\
${ }_{v} R 1_{A}^{T 6.5}$ & $3.5 \times 10^{4}$ & 7.5 & $D_{T 6.5}$ \\
${ }_{M}^{M} R 2_{A}^{T 6.42}$ & $3.5 \times 10^{4}$ & 7.5 & $D_{T 6.42}$ \\
${ }^{M} R 2_{A}^{T 6.42}$ & $1.0-2.5-3.5-6.5-8.5 \times 10^{5}$ & 7.5 & $D_{T 6.42}$ \\
${ }_{{ }^{\prime}} R 2_{A . K^{\prime}}^{T 6.42}$ & $3.5 \times 10^{4}$ & 1.5 & $D_{T 6.42}$ \\
\hline
\end{tabular}

for the diffusion coefficient:

$D_{T_{0}}=400 D_{\mathrm{He}}\left(T_{0}\right)\left[\frac{\rho\left(T_{0}\right)}{\rho}\right]^{3}$,

where $T_{0}$ is a free parameter that sets the depth of the maximum efficiency of the mixing depending on the value of the atomic diffusion coefficient $\mathrm{He}\left(D_{\mathrm{He}}\right)$. Initially introduced by Richer et al. (2000) to counteract an impact of atomic diffusion in AmFm stars that was too strong, we introduced it in our models with rotation to increase the surface Li depletion predicted on the MS and reproduce the observations for solar-type stars and for the Sun. For all the reference models, we adopted $\log T_{0}=6.42$ as calibrated by Paper I. We also present a set of models for the Hyades metallicity $([\mathrm{Fe} / \mathrm{H}]=+0.15 \mathrm{dex})$, where we increased this depth to $\log T_{0}=6.5$.

\subsection{Angular momentum evolution and rotation-induced mixing}

Stellar rotation was implemented in STAREVOL as described by Amard et al. $(2016,2019)$ and Paper I. We adopted the shellular rotation hypothesis developed by Zahn (1992), Maeder \& Zahn (1998), and Mathis \& Zahn (2004) to describe the transport of angular momentum and chemicals by meridional circulation, treated as an advective process for angular momentum, and turbulent shear (vertical and horizontal), treated as diffusive processes. We followed Eggenberger et al. (2012b, 2019b) and Spada et al. (2016) who introduced an additional parametric vertical viscosity $v_{\text {add }}$ in order to flatten the internal rotation profile as evidenced by helio- and asterosismology of low-mass stars. The transport of angular momentum hence obeys the following advection-diffusion equation:

$\rho \frac{\mathrm{d}}{\mathrm{d} t}\left(r^{2} \Omega\right)=\frac{1}{5 r^{2}} \frac{\partial}{\partial r}\left(\rho r^{4} \Omega U_{2}\right)+\frac{1}{r^{2}} \frac{\partial}{\partial r}\left(\left(v_{\mathrm{v}}+v_{\mathrm{add}}\right) r^{4} \frac{\partial \Omega}{\partial r}\right)$,

where $\rho, r, \Omega, U_{2}$, and $v_{\mathrm{v}}$ are the density, the radius, the angular velocity, the meridional circulation velocity, and the vertical shellular component of the turbulent viscosity, respectively. For the reference models, we adopted $v_{\text {add }}=3.5 \times 10^{4} \mathrm{~cm}^{2} \mathrm{~s}^{-1}$ in the entire radiative region. This value was calibrated in Paper I on the solar angular velocity profile provided by helioseismology. We also present a set of models for the Hyades metallicity $([\mathrm{Fe} / \mathrm{H}]=+0.15 \mathrm{dex})$ where we modified this value depending on the initial stellar mass $\left(v_{\text {add }}^{\prime}=3.5 \times 10^{5}, 1.0 \times 10^{5}, 2.5 \times 10^{5}\right.$, $6.5 \times 10^{5}$, and $8.5 \times 10^{5} \mathrm{~cm}^{2} \mathrm{~s}^{-1}$ for the $1.5,1.4,1.35,1.3$, and $1.2 M_{\odot}$ models, respectively). These values were kept constant during the entire evolution.

We explored two combinations of prescriptions for turbulence shear referred to as R1 and R2 as in Paper I; R1 includes prescriptions from Mathis et al. (2018) and Zahn (1992) for the horizontal diffusivity $D_{\mathrm{h}}$ and the vertical diffusivity $D_{\mathrm{v}}$, respectively, and R2 includes prescriptions from Zahn (1992) and Talon \& Zahn (1997) for $D_{\mathrm{h}}$ and $D_{\mathrm{v}}$, respectively. The detailed expressions of the four turbulent diffusion coefficients can be found in Appendix B of Paper I. We used the same parameters as in Paper I for the extraction of angular momentum at the stellar surface due to magnetised winds $(m=0.22, p=2.1, \chi=14$, $K=7.5 \times 10^{30} \mathrm{erg}$ unless otherwise indicated) according to the Matt et al. (2015) formalism. Models were computed for three values of the initial rotation period on the PMS: 1.6, 4.5, and 9.0 days, which are referred to as fast $\left({ }^{\mathbf{F}} R\right)$, median $\left({ }^{\mathbf{M}} R\right)$, and slow $\left({ }^{\mathbf{S}} R\right)$ rotating models, respectively. The disc coupling timescale was set at $\tau_{\text {disc }}=2.5$ Myrs for the fast rotators and at $\tau_{\text {disc }}=5$ Myrs for the median and the slow rotators, in agreement with Gallet \& Bouvier (2015) and Amard et al. (2019).

To summarise, the best models for solar-type stars that we developed in Paper I include the self-consistent treatment of physical processes as well as parametrised additional transports for angular momentum and chemicals as summarised in Table 2. The parameters are strongly constrained by observations (see Col. 3 Table 2) and cannot be varied freely. In the present study, we explore a possible variation in the efficiency of these additional processes with metallicity and initial mass.

\section{Model predictions for $\mathrm{Li}, \mathrm{Be}$, and surface rotation - The Hyades and Praesepe test case}

In this section, we explore the predictions of model ${ }_{v} R 1_{A}^{T 6.42-6.5}$ over a range of masses and metallicities for three different rotation rates. We recall that this model was developed for the specific case of solar-type stars in Paper I. We discuss the general impact of mass and metallicity on Li depletion, and then we compare the predictions for the behaviour of surface $\mathrm{Li}, \mathrm{Be}$, and rotation rates to the observations in the Hyades and Praesepe, and to the predictions of classical models and of model ${ }_{v}^{M} R 2_{A}^{T 6.42}$ computed with different prescriptions for rotation-induced turbulence (see Table 3 ).

\subsection{Impact of metallicity and mass on Li evolution}

Figure 1 shows the evolutionary tracks in the HertzsprungRussell diagram (PMS and MS) and the predicted surface Li abundance as a function of $T_{\text {eff }}$ for a selection of models (all computed with median rotation). We see the well-known impact of varying the mass and the metallicity on the evolution tracks, and the global consequence on Li depletion. When metallicity decreases for a given mass, or when the mass increases for a given metallicity, the model is hotter and brighter. As a consequence, its convective envelope retracts more rapidly at the beginning of the Hayashi track on the PMS, and it is thinner on the MS. Hence, its base is more distant from the layers where $\mathrm{Li}$ 


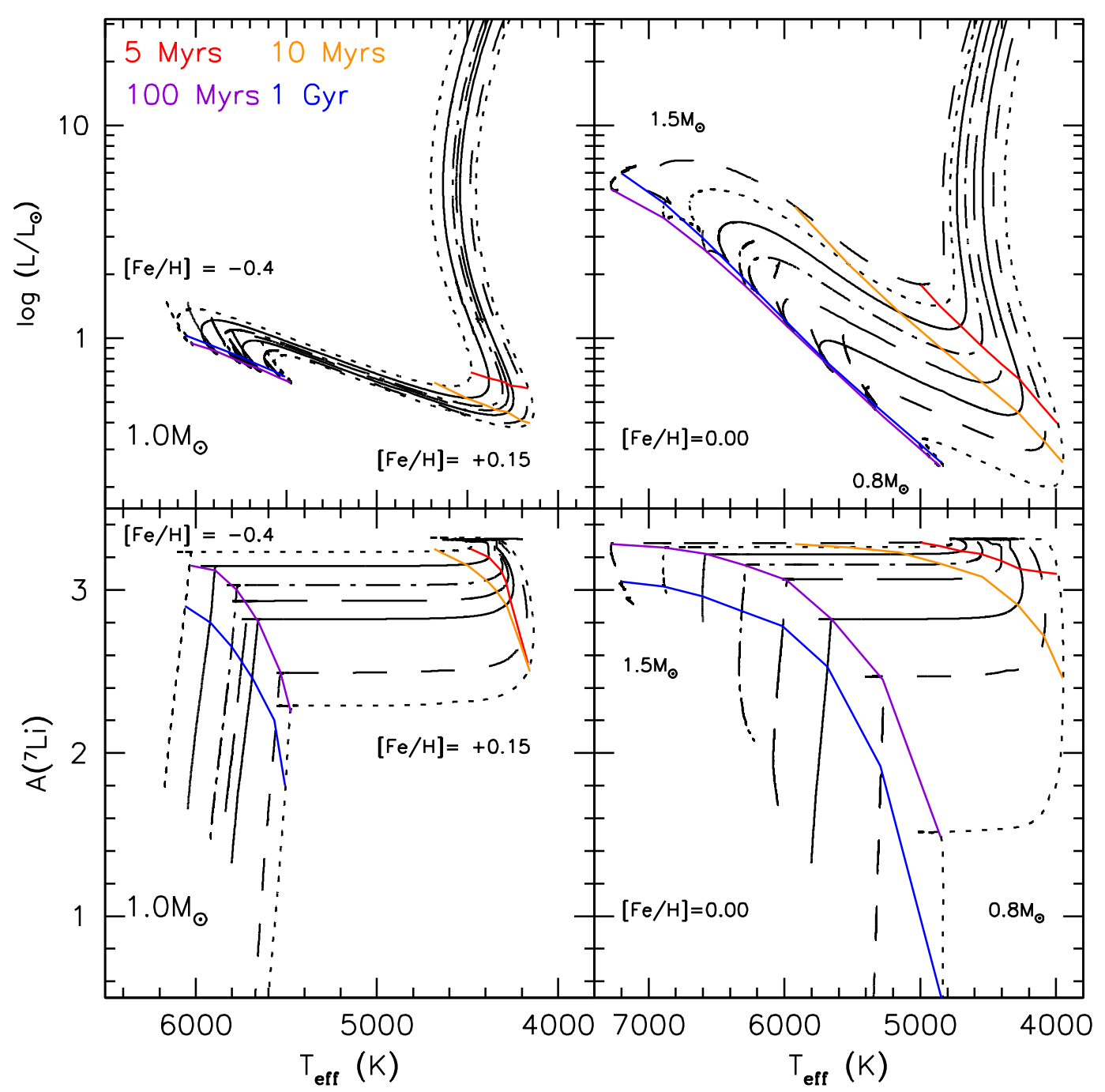

Fig. 1. Top: evolutionary tracks in the Hertzsprung-Russell diagram of ${ }_{v}^{M} R 1_{A}^{T 6.42}$ models of $1.0 M_{\odot}$ computed for seven values of [Fe/H] (left) and of different masses for $[\mathrm{Fe} / \mathrm{H}]=0$ (right). Bottom: surface $\mathrm{Li}$ abundance for the corresponding models. The coloured-lines connect points of the same age.

is burning, which overall leads to lower Li depletion, although the quantitative details depend on the efficiency of the transport process(es) that connect(s) the base of the convective envelope to the Li-free region (see below).

In all the models shown in Fig. 1, Li decreases along two successive episodes, first on the PMS, and later along the MS. The PMS depletion episode is first due to Li burning in the convective envelope at the beginning of the Hayashi track and later to overshoots that are both dependent on the size of the convective envelope and its timescale for retraction along the PMS. As a consequence, PMS depletion is minute for stars with $\mathrm{ZAMS}^{5}$ $T_{\text {eff }}$ higher than $\sim 6500 \mathrm{~K}$, and it increases with decreasing stellar mass and increasing metallicity.

The MS depletion episode is due to the combined effects of rotation-induced mixing and parametric turbulence. The first process is more efficient in cooler stars (i.e., less massive at a given metallicity, or more metal-rich at a given mass), which undergo more significant extraction of angular momentum by their magnetised winds (see e.g., Fig. 6 in Amard et al. 2019). Similar mass and metallicity dependencies exist in the case of the second process because in all of our models we assume, due

\footnotetext{
5 Zero age main sequence.
}

to Eq. (2), the same value for the free parameter $T_{0}$ which is closer to the temperature of the base of the convective envelope in less massive or more metal-rich stars. In summary, overall $\mathrm{Li}$ depletion is stronger in less massive, more metal-rich stars.

\subsection{The Hyades and Praesepe}

Here we focus on the well-studied Hyades and Praesepe for which $\mathrm{Li}, \mathrm{Be}$, and surface rotation data are available. These two open clusters are close in age $(0.72 \mathrm{Gyr}$ and $0.75 \mathrm{Gyr}$, respectively) and metallicity $([\mathrm{Fe} / \mathrm{H}]=+0.13 \pm 0.05$ and $+0.16 \pm$ 0.08 dex, respectively) according to the references quoted in Table 1.

Figure 2 shows the observed $\mathrm{Li}$ abundances and rotation velocities $(V \sin i)$ as a function of the effective temperature from Cummings et al. (2017). Both clusters exhibit similar patterns, namely the so-called Li dip between $\sim 6400$ and $6800 \mathrm{~K}$, the $\mathrm{Li}-T_{\text {eff }}$ decrease in G-type stars on the cool side of the dip, and the well-know break in rotation velocity $(\approx 6400-6500 \mathrm{~K})$ for dwarf stars later than the F4 spectral type (Schatzman et al. 1959; Schatzman 1962; Kraft 1967; Boesgaard 1987). In the same figure, we show the predictions at 0.75 Gyr of the classical models, and of the complete models ${ }_{v} R 1_{A}^{T 6.42}$ and of ${ }_{v} R 1_{A}^{T 6.5}$ 
T. Dumont et al.: Lithium depletion and angular momentum transport in F-type and G-type stars in Galactic open clusters

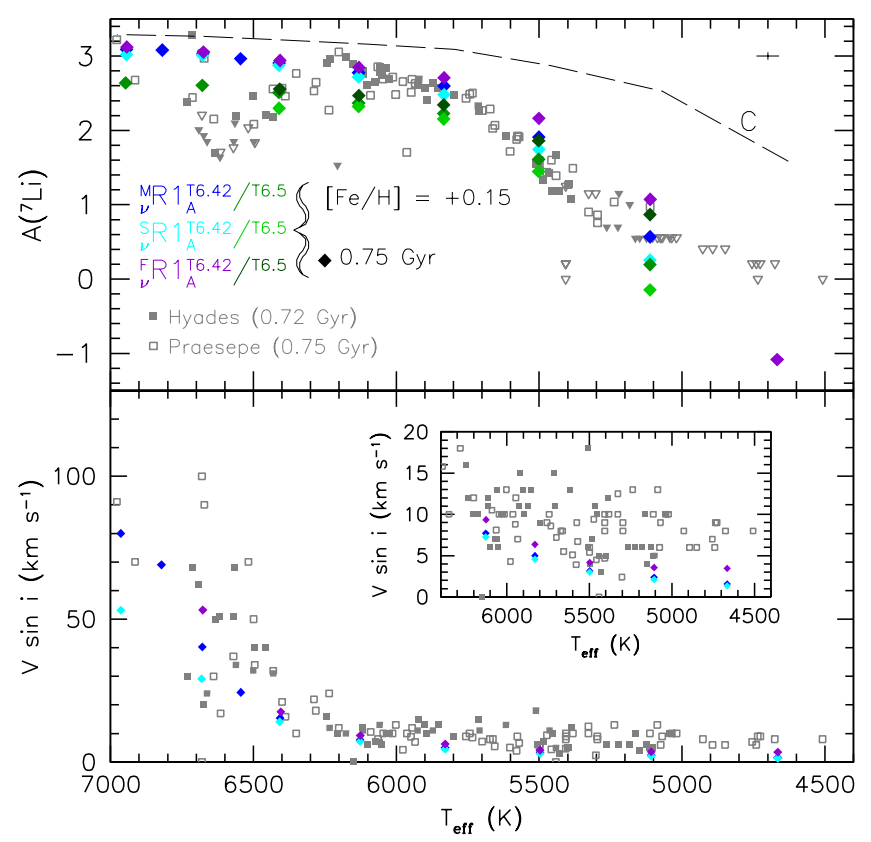

Fig. 2. Top: Li surface abundance versus $T_{\text {eff }}$ in the Hyades (filled squares) and Praesepe (open squares) from Cummings et al. (2017); downward triangles are for upper limits. Comparison to the predictions at $0.75 \mathrm{Gyr}$ (diamonds) of models with $[\mathrm{Fe} / \mathrm{H}]=+0.15$ computed under several assumptions: classical models ( $C$; dashed black line) and complete models ${ }_{v} R 1_{A}^{T 6.42}$ (cyan, blue, and violet diamonds for slow, median, and fast initial rotation velocities, respectively) and ${ }_{v} R 1_{A}^{T 6.5}$ (light green, green, and dark green diamonds for slow, median, and fast initial rotation velocities, respectively). Bottom: $V \sin i$ versus $T_{\text {eff }}$ in the Hyades (filled squares) and Praesepe (open squares) from Cummings et al. (2017). Comparison to the surface rotation velocity predictions of model ${ }_{v} R 1_{A}^{T 6.42}$ at $0.75 \mathrm{Gyr}$. Insert: Zoom on the cool side of the Kraft rotation break including model predictions for 0.8-1.2 $M_{\odot}$ stars.

models computed with $[\mathrm{Fe} / \mathrm{H}]=+0.15$ for three different initial rotation velocities (slow, median, and fast) for masses between 0.8 and $1.5 M_{\odot}$ (mass step $0.1 M_{\odot}$ ), and for a median initial rotation velocity for the 1.35 and $1.45 M_{\odot}$ models.

As has been long known in the literature, and as shown in Fig. 2, classical models (model C) that account solely for convection predict $\mathrm{Li}$ depletion on the PMS only, when the size of the convective envelope is large enough to reach the Li-burning temperatures along the Hayashi track (see Sect. 4.1). While the predicted mass and $T_{\text {eff }}$ dependence of the $\mathrm{Li}$ abundance after the PMS depletion is similar to the observed pattern for G-type stars, the predicted surface $\mathrm{Li}$ abundances are too high compared to the observations in the Hyades and Praesepe, as well as in the other clusters of different ages considered in this work (see Sect. 5).

Models ${ }_{v} R 1_{A}^{T 6.42}$ and ${ }_{\nu} R 1_{A}^{T 6.5}$ predict rotation velocities that account for the observed $V \sin i$ trends well (including the position in effective temperature of the Kraft break) in both open clusters, thanks to the extraction of angular momentum due to magnetised winds (see also Fig. 5). However, on the cool side of the Kraft rotation break (see insert in Fig. 2), models reproduce the lower observational envelope well, but not the entire observed spread. It is mainly the case for the lowest stellar masses where the parametric viscosity is more efficient.

Models ${ }_{v} R 1_{A}^{T 6.42}$ and ${ }_{v} R 1_{A}^{T 6.5}$ also provide a very good fit to the Li data in G-type stars on the cool side of the Li dip where angular momentum extraction is maximal, that is, for stellar masses lower than $1.3 M_{\odot}$ for the metallicity of these clusters. At the age of the Hyades and Praesepe, the increased (with respect to the classical model C) Li depletion is mainly due to penetrative convection (Eq. (1)) along the PMS, and only slightly due to turbulent mixing at the beginning of the MS. As discussed in Paper I, the Augustson \& Mathis (2019) expression for penetrative convection leads to larger Li depletion for slow rotators than for fast rotators because of the influence of the rotation rate on the depth of the overshoot via the ratio $v / v_{0}$ in Eq. (1). This anticorrelation with the rotation rate, which has long been observed (e.g., Bouvier 2008; Bouvier et al. 2018; Arancibia-Silva et al. 2020), is obtained here for the first time for the entire mass range considered at the Hyades and Praesepe age ${ }^{6}$. In addition, the predicted $\mathrm{Li}$ spread induced by the different initial rotation rates assumed here (Sect. 3.4) is amplified in lower mass stars where the base of the convective envelope is deeper and closer to the Li burning layers, which also leads to more efficient parametric turbulence on the lower mass end. For this later process, we present model predictions for two values of the parameter $T_{0}$ from Eq. (2), that is, $\log T_{0}=6.42$ as calibrated in the Sun and $\log T_{0}=6.5$ which better fits the data spread in cool stars of the Hyades and Praesepe. Overall, our complete model ${ }_{v} R 1_{A}^{T 6.42-6.5}$ reproduces fairly well the $\mathrm{Li}-T_{\text {eff }}-V \sin i$ trend and the observed Li dispersion on the cool side of the Li dip (i.e., G-type and late F-type stars). In this $T_{\text {eff }}$ domain, it also accounts for the Be data in the Hyades and Praesepe, as shown in Figs. 3 and 4.

However, models ${ }_{v} R 1_{A}^{T 6.42-6.5}$ predict little Li depletion (both on the PMS and the MS at the ages of the Hyades and Praesepe) for stars more massive than $1.3 M_{\odot}$ that have small convection zones that are vanishing on the MS and which undergo a negligible angular momentum braking. As a consequence, it does not reproduce the depth of the $\mathrm{Li} \mathrm{dip,} \mathrm{nor} \mathrm{the} \mathrm{Li} \mathrm{rise} \mathrm{on} \mathrm{the} \mathrm{cool} \mathrm{edge}$ of the dip. Around $6600 \mathrm{~K}$, where many stars only have Li upper limits, the maximum Li depletion predicted reaches only $\sim 1$ dex in the case where we assume slightly deeper parametric turbulence (i.e., $\log T_{0}=6.5$ ). This low depletion mainly results from the choice of prescriptions for the horizontal and vertical diffusivities $D_{\mathrm{h}}$ and $D_{\mathrm{v}}$ (taken here from Mathis et al. 2018 and Zahn 1992, respectively; see Sect. 3.4). This is in contrast with previous studies on the hot edge of the Li dip (Palacios et al. 2003), where the use of the prescriptions by Zahn (1992) and Talon \& Zahn (1997) for $D_{\mathrm{h}}$ and $D_{\mathrm{v}}$, respectively, led to a good match between model predictions and observations. As can be seen in Fig. 3, much stronger $\mathrm{Li}$ and Be depletion is indeed obtained in the model ${ }_{v}^{M} R 2_{A}^{T 6.42}$ where we used the same prescriptions as in that earlier paper. As already evidenced in Paper I for the solar case, this combination however leads to $\mathrm{Li}$ depletion that is too strong in F- and G-type stars for the value of $v_{\text {add }}$ adopted in that paper and here $\left(3.5 \times 10^{4} \mathrm{~cm}^{2} \mathrm{~s}^{-1}\right)$. Additionally, reducing the torque parameter $\mathrm{K}$ for the wind from Matt et al. (2015), from $7.5 \times 10^{30}$ erg to $1.5 \times 10^{30} \mathrm{erg}$, improves the comparison on the hot edge of the Li dip and of the Be dip, but not on the cool edge as can be seen in Figs. 3 and 4 for model ${ }_{v}^{M} R 2_{A . K^{\prime}}^{T 6.42}$.

Talon \& Charbonnel (1998) reached similar conclusions as Palacios et al. (2003), using the same turbulence prescriptions $\left(D_{\mathrm{h}}\right.$ and $D_{\mathrm{v}}$ from Zahn 1992 and Talon \& Zahn 1997, respectively). These authors interpreted the rise in $\mathrm{Li}$ on the cool

\footnotetext{
6 This anti-correlation was initially obtained by Somers \& Pinsonneault (2015) who invoked a radius inflation dependent on the rotation velocity to explain the anti-correlation at the Pleiades age. However, even though it has been confirmed in the Pleiades (Somers \& Pinsonneault 2016; Somers $\&$ Stassun 2017), the effect of this process still needs to be clarified at older ages than Pleiades and for different metallicities (e.g., Jaehnig et al. 2019; Jackson et al. 2019).
} 

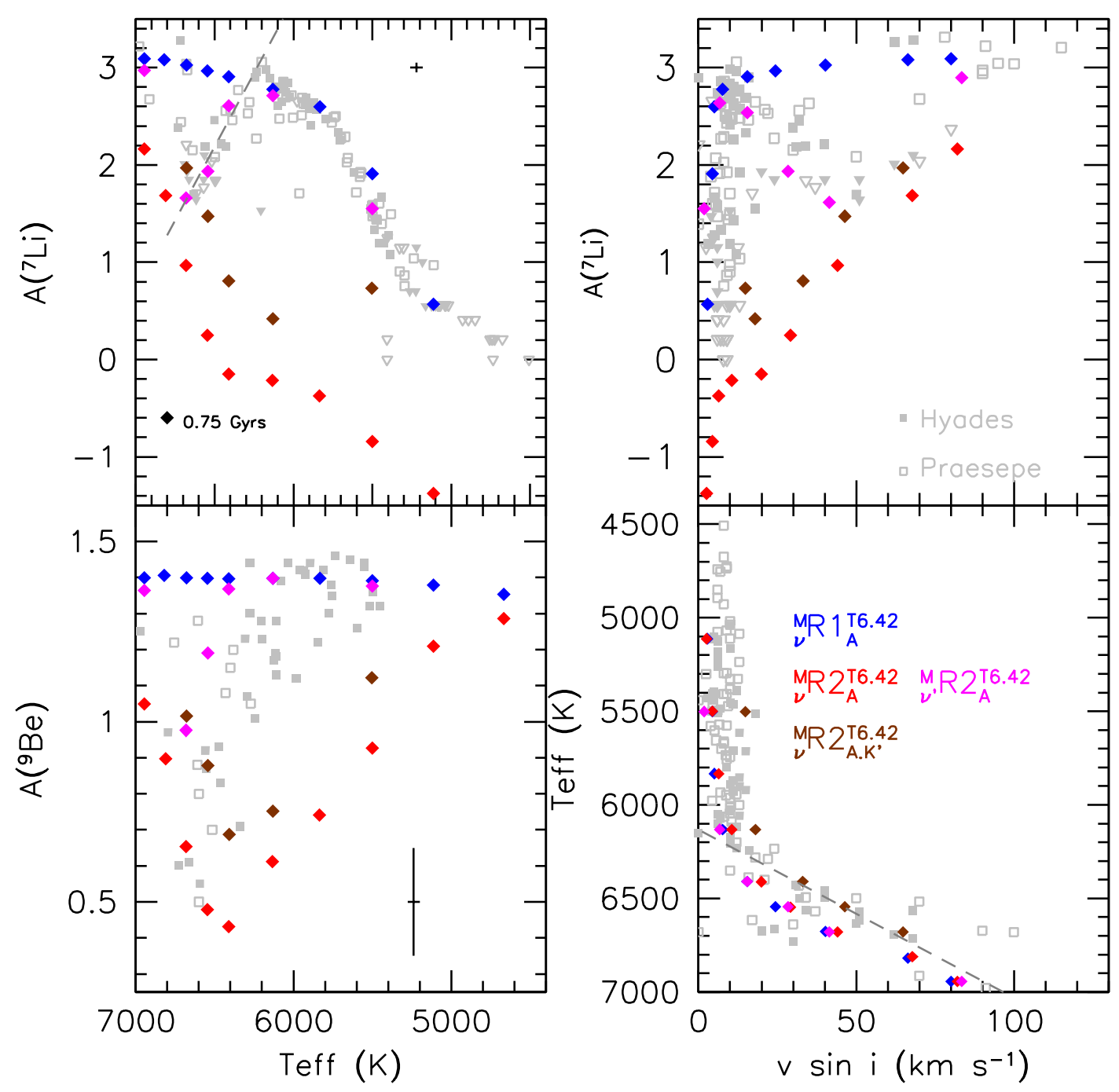

Fig. 3. Comparison of the Li, Be, and $V \sin i$ observations in the Hyades and Praesepe (filled and open grey squares, respectively; triangles are for abundance upper limits; see Table 1 for references) with the predictions of models ${ }_{v}^{M} R 1_{A}^{T 6.42}$ (blue diamonds) and ${ }_{v^{\prime}}^{M} R 2_{A}^{T 6.42}$ for different values of $v_{\text {add }}^{\prime}$ and K (colour-coded, see also Table 3). Model predictions are shown at 0.75 Gyr. The grey dashed line represents the empirical relation in the cold edge of the dip obtained by Cummings et al. (2017).

edge of the dip as the signature of the appearance of a process that transports angular momentum more efficiently than meridional circulation and turbulent shear. Talon \& Charbonnel $(2003,2005)$ then showed that the generation of internal gravity waves (hereafter IGW) by the stellar convective envelope becomes efficient inside the Li dip and increases on its cool edge. In their model, the maximum efficiency of the IGWs in terms of angular momentum transport is expected at $T_{\text {eff }}$ around 5800$5900 \mathrm{~K}$, before it decreases in cooler stars (see Fig. 6 of Talon \& Charbonnel 2003 and Fig. 4 of Talon \& Charbonnel 2004). Last but not least, their model also accounts for the internal solar rotation (Charbonnel \& Talon 2005). It thus has the proper mass and $T_{\text {eff }}$ dependence to be the required transport mechanism involved, contrary to other processes that could also potentially transport angular momentum (e.g., the Taylor-Spruit dynamo Spruit 2002; Eggenberger et al. 2005, 2010).

Following this theoretical trend for IGWs, we computed the ${ }_{v^{\prime}}^{M} R 2_{A}^{T 6.42}$ of various masses assuming a $T_{\text {eff }}$ dependence of the parametric viscosity $v_{\text {add }}^{\prime}$ (increasing value with decreasing mass on the cool edge of the Li dip) in order to mimic a transport of angular momentum as predicted for low-mass stars. Although the use of a uniform and constant parametric viscosity within the radiative layers leads to a very different angular momentum profile than that shaped by IGW (compare Fig. 7 with Fig. 1 of Charbonnel \& Talon 2005), higher values of $v_{\text {add }}^{\prime}$ mimic a more efficient internal transport of angular momentum compared to that driven by meridional circulation and turbulent shear (see Fig. 7 which is discussed in detail in Sect. 7), hence reducing their efficiency for the transport of chemicals. The $v_{\text {add }}^{\prime}$ values required to reproduce the shape of the Li dip are $3.5 \times 10^{5} \mathrm{~cm}^{2} \mathrm{~s}^{-1}$ and $1.0 \times 10^{5} \mathrm{~cm}^{2} \mathrm{~s}^{-1}$ for the 1.5 and $1.4 M_{\odot}$ models, respectively, on the hot edge of the $\mathrm{Li}$ dip, and $2.5 \times 10^{5}, 6.5 \times 10^{5}$, and $8.5 \times 10^{5} \mathrm{~cm}^{2} \mathrm{~s}^{-1}$ for the $1.35 M_{\odot}, 1.3 M_{\odot}$, and $1.2 M_{\odot} \bmod -$ els, respectively, on the cool edge of the dip. On the other hand, the $\mathrm{Li}$ abundance in the $1.0 \mathrm{M}_{\odot}$ model is well reproduced for $v_{\text {add }}^{\prime}=2.5 \times 10^{5} \mathrm{~cm}^{2} \mathrm{~s}^{-1}$. However for this mass, it leads to a reduction that is too strong of the surface velocity (see Figs. 5 and 7), which makes it hard to reconcile with $P_{\text {rot }}$ measurements in the Hyades and Praesepe. As shown in Figs. 3 and 4, these assumptions explain the Be plateau in cool stars, within the observational uncertainties. However, the ${ }_{v^{\prime}}^{M} R 2_{A}^{T 6.42}$ models do not account for the large Be depletion in the Be dip nor in its cool edge. This conclusion holds for all the initial rotation rates explored here. 
T. Dumont et al.: Lithium depletion and angular momentum transport in F-type and G-type stars in Galactic open clusters

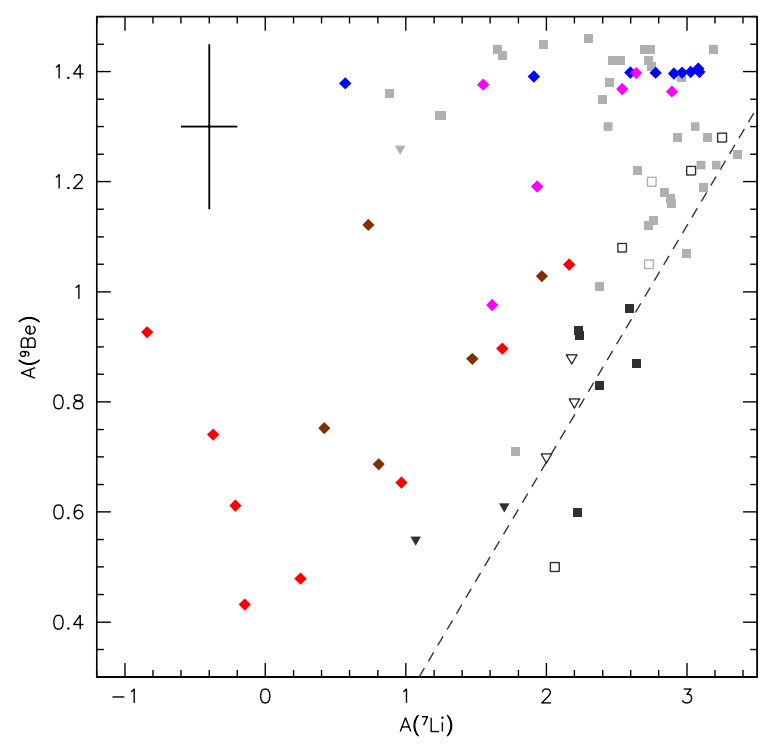

Fig. 4. Comparison of the models ${ }_{v}^{M} R 1_{A}^{T 6.42}$ (blue diamonds), ${ }_{v}^{M} R 2_{A}^{T 6.42}$ (red diamonds), ${ }_{v}^{M} R 2_{A . K^{\prime}}^{T 6.42}$ (brown diamonds), and ${ }_{\gamma^{\prime}}^{M} R 2_{A}^{T 6.42}$ (magenta diamonds) with observations of surface $\mathrm{Li}$ and $\mathrm{Be}$ in the Hyades (filled squares) and Praesepe (open squares) open clusters. Downward triangles are for $\mathrm{Li}$ upper limits. Stars corresponding to the $\mathrm{Li}$ dip $\left(6400 \mathrm{~K}<T_{\text {eff }}<6800\right)$ are represented in dark grey and stars outside of the Li dip are represented in light grey. Observations are directly extracted from Boesgaard et al. (2004b, 2016) without any modification (no NLTE correction for Li abundances). The dark grey dashed line represents the linear correlations found by Boesgaard et al. (2020) with $A(\mathrm{Be})=0.43 \times A(\mathrm{Li})-0.17$ for stars in the cold edge of the Li dip.

It thus appears very difficult to reconcile the $\mathrm{Li}, \mathrm{Be}$, and surface rotation rate constraints in the test case of the Hyades and Praesepe. While the ${ }_{v} R 1_{A}^{T 6.42}$ models account for the surface rotation well over the entire mass domain explored, and for the $\mathrm{Li}$ and $\mathrm{Be}$ abundances in G-type stars, they fail to explain the $\mathrm{Li}$ and $\mathrm{Be}$ dips in these two clusters. On the other hand, ${ }_{\gamma^{\prime}}^{M} R 2_{A}^{T 6.42}$ models with the parametric viscosity for the transport of angular momentum, dependent on the stellar mass of the star and adjusted to fit the $\mathrm{Li}$ abundances along the entire mass range, predict a Be dip that is not as deep as the one observed, and they lead the $1 M_{\odot}$ model to rotate too slowly at the age of the two clusters $(0.72-0.75$ Gyr). In Sect. 5 we evaluate the respective compatibility of the models ${ }_{v} R 1_{A}^{T 6.42}$ and ${ }_{\gamma^{\prime}}^{M} R 2_{A}^{T 6.42}$ with the surface $\mathrm{Li}$ and rotation rates in a sample of open clusters, before discussing in Sect. 6 the impact of the different assumptions for the transport of angular momentum on the internal rotation profiles of the models in the light of asteroseismic constraints. In Sect. 5, we provide a summary of the successes and difficulties of the different assumptions, and we discuss their meaning in terms of the uncertainties of the different processes involved.

\section{Model predictions for $\mathrm{Li}$ and surface rotation - Comparison to open clusters of different ages and metallicities}

In this section, we compare the predictions of models ${ }_{\nu} R 1_{A}^{T 6.42}$ and ${ }_{v^{\prime}}^{M} R 2_{A}^{T 6.42}$ to rotation rates and surface $\mathrm{Li}$ abundances for a sample of open clusters with different ages and metallicities (see Table 1). Be cannot be used here, as there are not enough available data for clusters other than the Hyades and Praesepe. In the ${ }_{v} R 1_{A}^{T 6.42}$ case, we computed models for the actual $[\mathrm{Fe} / \mathrm{H}]$ of the individual clusters and for three initial rotation rates (slow, median, and fast), while in the ${ }_{v^{\prime}}^{M} R 2_{A}^{T 6.42}$ case, we used the models at the metallicity of the Hyades and Praesepe $([\mathrm{Fe} / \mathrm{H}]=+0.15 \mathrm{dex})$, and for median rotation discussed previously.

The values of A(Li), $T_{\text {eff }}$, and $P_{\text {rot }}$ predicted by the ${ }_{v}^{M} R 1_{A}^{T 6.42}$ model are given in Appendix A for the different masses and cluster ages.

\section{1. $P_{\text {rot }}$ versus mass}

In Fig. 5 we compare the predicted surface rotation periods of the ${ }_{v} R 1_{A}^{T 6.42}$ models with relevant $[\mathrm{Fe} / \mathrm{H}]$ to observational data for a sample of 12 open clusters at different ages. We added the ${ }_{v^{\prime}}^{M} R 2_{A}^{T 6.42}$ model predictions at the $[\mathrm{Fe} / \mathrm{H}]$ of the Hyades for $\alpha \mathrm{Per}$, Hyades, Praesepe, and NGC 6819. The masses of the individual cluster stars were determined by isochrone fitting in the original papers quoted in Table 1 for the $P_{\text {rot }}$. Godoy-Rivera et al. (2021) have studied the difference in mass estimates that can be derived from using different families of isochrones and they show that it is quite small $\left(\Delta M \approx 0.05 M_{\odot}\right)$.

We confirm the conclusions reached in previous studies (e.g. Krishnamurthi et al. 1997; Allain 1998; Irwin \& Bouvier 2009; Amard et al. 2016, 2019): the evolution of the rotation period can be divided in several phases that our models succeed to predict. Firstly, the large dispersion at very young ages for all masses (NGC 2362 to IC 2391, first row of Fig. 5) results in our models from the assumption of constant surface angular velocity as long as disc-locking is efficient. Secondly, the progressive slow down of first, slow and median, and finally fast rotators of decreasing mass in clusters of increasing age (from $\alpha$ Per to M 37, second row of Fig. 5) is very well reproduced by our models as the result of the secular evolution of the radius along the PMS and the efficient wind braking on the early MS. Finally, for clusters older than Praesepe ( $\approx 750$ Myrs), we observe the convergence of the three families of rotators into one single sequence recovering the observational law by Skumanich (1972). In addition, the sequence presents a change in slope around $\mathrm{M} \approx 1.2 M_{\odot}$ in the $P_{\text {rot }}$ versus mass diagram (last row of Fig. 5), resulting from the magnetic braking by the stellar winds. This change in slope corresponds to the Kraft break and to the mass domain where the convective envelope becomes very thin. In the Hyades and Praesepe, we also show the predictions of the model ${ }_{\gamma^{\prime}}^{M} R 2_{A}^{T 6.42}$ introduced to fit the cool edge of the Li dip (see Sect. 4.2). The predicted rotation periods for models more massive than $1.2 M_{\odot}$ are fully compatible with observations, while the $1 M_{\odot}$ median rotator model is far too slow, as already discussed in Sect. 4.2. As in Amard et al. (2019), who use the same prescriptions for horizontal and vertical turbulence and for the braking law as in model ${ }_{v} R 1_{A}^{T 6.42}$ (with a parameter $K=7.0 \times 10^{30} \mathrm{erg}$ instead of $7.5 \times 10^{30} \mathrm{erg}$ ) but who do not include the parametric viscosity for the internal transport of angular momentum, our model predictions for the older clusters present a larger negative slope on the lower mass side, with the lower mass models spinning slower than the observed stars. For the more massive stars in NGC 6819, the extraction of angular momentum, which is directly linked to the depth of the convective envelope, becomes inefficient in the models, which spin faster than the observed stars. Finally, for the Pleiades we obtain a poor agreement with observations for the lowest stellar masses $\left(0.8\right.$ and $\left.0.9 M_{\odot}\right)$ compared to what we get for all the other clusters and to what was obtained in Amard et al. (2019). This is essentially due to the difference in age adopted for this cluster in both studies (125 Myrs from Barrado y Navascués et al. 2004 in Amard et al. 2019 instead of 87 Myrs as assumed here), as shown in Fig. 5 where the orange 


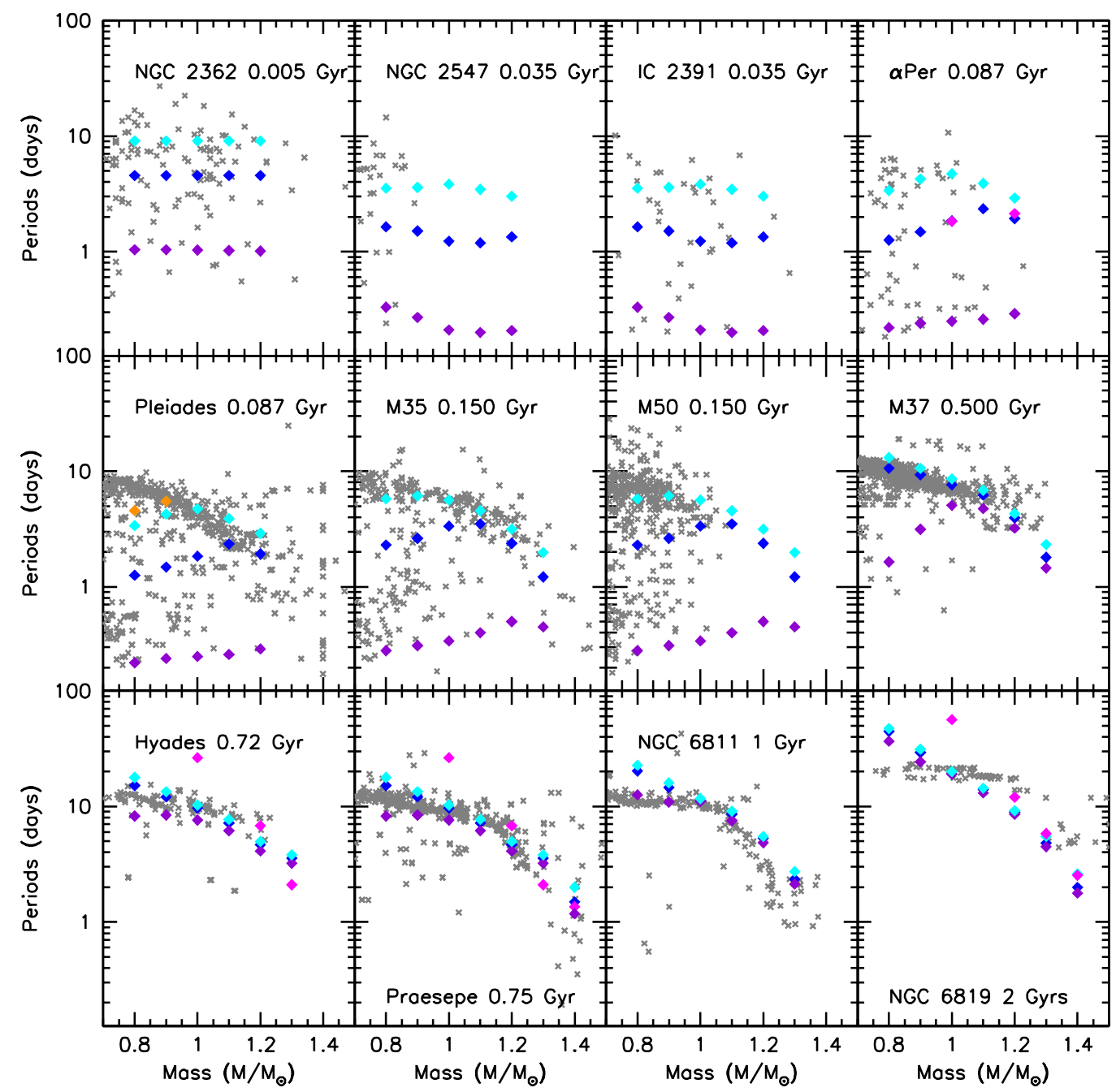

Fig. 5. Surface rotational period versus mass. Comparison of the models ${ }_{v} R 1_{A}^{T 6.42}$ (diamonds) at three different initial velocities: slow (cyan), median (blue), and fast (violet) with the observations for open clusters at different ages and metallicities. The orange diamond for Pleiades corresponds to a slow rotating model at $125 \mathrm{Myrs}$. In the case of $\alpha$ Per, Hyades, Praesepe, and NGC 6819, we also show the predictions of the ${ }_{v^{\prime}}^{M} R 2_{A}^{T 6.42}$ models (magenta diamonds). Observations' references are reported in Table 1.

diamonds are the predictions of our models at 125 Myrs, which better account for the data.

To summarise, the comparison between the predictions of our ${ }_{v} R 1_{A}^{T 6.42}$ and ${ }_{v^{\prime}}^{M} R 2_{A}^{T 6.42}$, and the observational data for the rotation period is rather satisfactory, although some peculiar features remain difficult to reproduce without further adjustments. This work confirms the global theoretical behaviour obtained by Amard et al. $(2016,2019)$ despite the fact that we included an additional diffusive source of transport of angular momentum in the present study.

\subsection{Lithium $-T_{\text {eff }}$}

In Fig. 6, we compare the predictions of models ${ }_{v} R 1_{A}^{T 6.42}$ for the surface $\mathrm{Li}$ abundances to data within the cluster sample (see Sect. 2 and Table 1), and we report the predictions of models ${ }_{v^{\prime}}^{M} R 2_{A}^{T 6.42}$ discussed in Sect. 4 for the most metal-rich clusters ( $\alpha$ Per, Hyades, Praesepe, and NGC 6819). Importantly, over the entire mass and metallicity range explored, the faster the initial rotation velocity, the lower the predicted Li depletion. As already discussed in Sect. 4.2, this is due to penetrative convection acting on the PMS, which we simulated with the Augustson \& Mathis (2019) prescription.
The Li- $T_{\text {eff }}$ relation for G-type stars is well reproduced by models ${ }_{v} R 1_{A}^{T 6.42}$ at all ages from the youngest (IC 2602 and IC 2391) to the oldest cluster (M67) with solar or super-solar metallicities. For the young clusters with subsolar metallicity (M35, NGC 6633, and UMa), the cooler models, corresponding to lower mass stars (see Table A.1), however predict larger A(Li) values than observed. In these models, the convective envelope is thinner due to the lower metallicity and the penetrative convection, which is the main process responsible for the Li depletion on the PMS and it is less efficient than at higher metallicity. This can be directly seen when comparing the panels showing NGC 6633 and UMa clusters as well as the Hyades and Praesepe clusters, which have similar ages but different metallicities. This suggests a stronger metallicity dependence of the Li depletion in cool stars compared to that predicted. Data for the cooler stars in the most metal-poor and oldest cluster (NGC 2243) would be required to confirm this trend at older ages.

We see in Fig. 6 that the Li dip is not present in the youngest clusters, and that its depth increases with time along the MS (see also Wallerstein et al. 1965; Boesgaard \& Tripicco 1986; Hobbs \& Pilachowski 1986; Soderblom et al. 1993b; Balachandran 1995; Steinhauer \& Deliyannis 2004; 


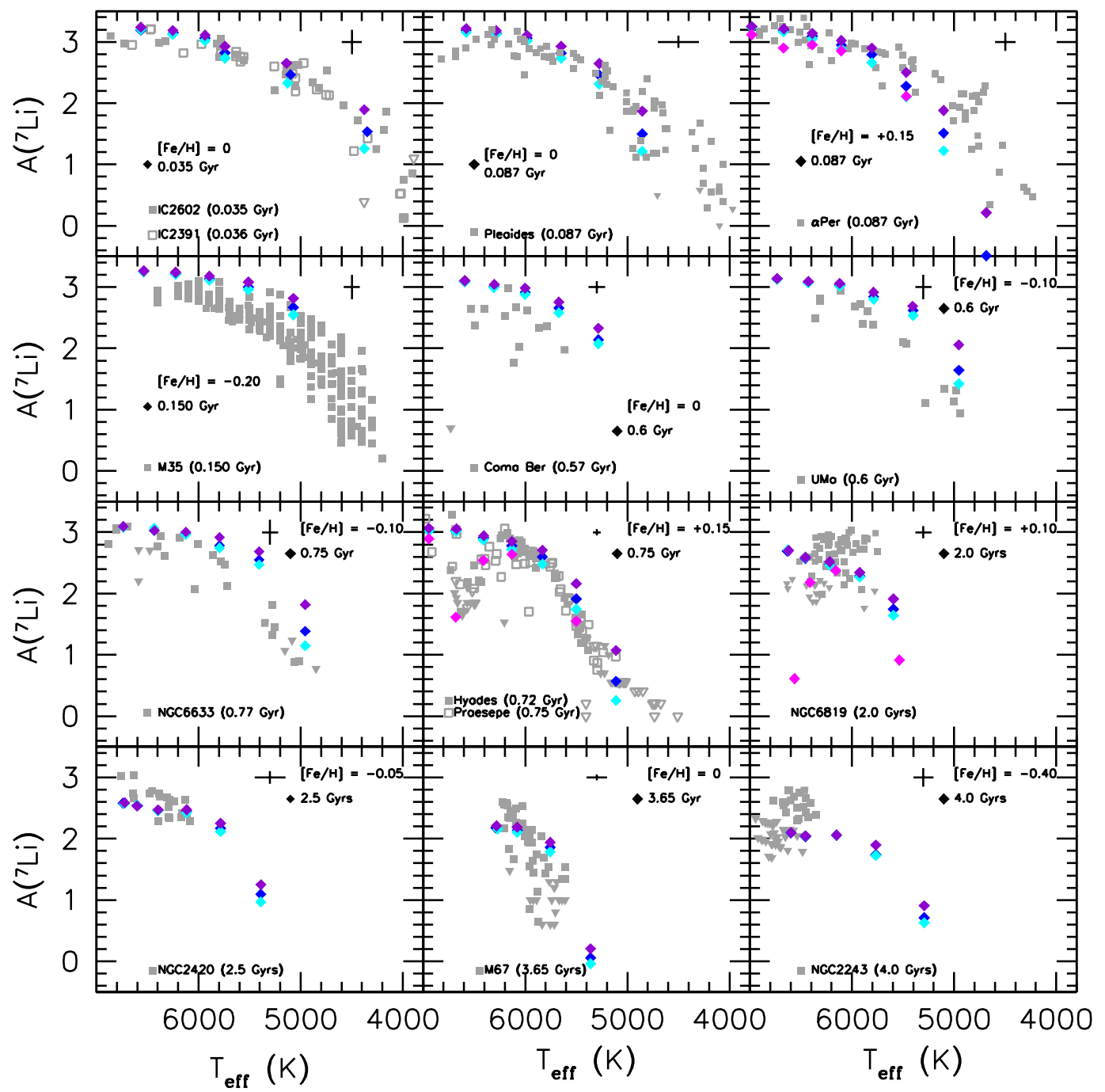

Fig. 6. Li surface abundance versus $T_{\text {eff }}$ in open clusters of different ages and $[\mathrm{Fe} / \mathrm{H}]$ (see Table 1 for references; grey squares and triangles are abundance determinations and upper limits, respectively). The typical observation errorbars from the original papers are indicated by a cross in each panel. Coloured diamonds are the predictions of the models at the age and metallicity of the corresponding clusters. The ${ }_{v} R 1_{A}^{T 6.42}$ models are shown for three initial velocities (slow, median, and fast are the cyan, blue, and violet diamonds, respectively), and models ${ }_{v^{\prime}}^{M} R 2_{A}^{T 6.42}$ for median rotation only (magenta diamonds). Models from left to right in each panel correspond to masses between $1.5 M_{\odot}$ (warmer) and $0.8 M_{\odot}$ (cooler).

Anthony-Twarog et al. 2009; Cummings et al. 2012; Boesgaard et al. 2016). As already discussed in Sect. 4.2, model ${ }_{v} R 1_{A}^{T 6.42}$ predicts too little Li depletion in the dip, while ${ }_{\gamma^{\prime}}^{M} R 2_{A}^{T 6.42}$ models (computed only for $[\mathrm{Fe} / \mathrm{H}]=+0.15 \mathrm{dex}$ ) reproduce both the depth and the shape of the Li dip in clusters with super-solar metallicities and with ages between 720 Myrs (Hyades) and 2 Gyrs (NGC 6819). For the old low metallicity NGC 2243 cluster, the cold edge of the Li dip is shifted towards slightly higher $T_{\text {eff }}$ compared to the younger higher metallicity clusters (Cummings et al. 2012; François et al. 2013). Since models ${ }_{v} R 1_{A}^{T 6.42}$ lie below the observational points on the cold edge of the Li dip, we expect that model ${ }_{v^{\prime}}^{M} R 2_{A}^{T 6.42}$, assuming a similar dependence between $v_{\text {add }}^{\prime}$ and $T_{\text {eff }}$ as assumed to fit the Hyades and Praesepe, would predict an even stronger Li depletion and would be incompatible with the data of NGC 2243.

We thus reach similar conclusions as in Sect. 4.2, for a relatively large range of metallicities and ages. Model ${ }_{\nu} R 1_{A}^{T 6.42}$ initially developed to reproduce surface and internal constraints for solar-type stars nicely explains the general trend of the data for both the rotation and Li behaviours over the mass and the metallicity ranges probed in this study, except for the $\mathrm{Li}$ dip. On the other hand, model ${ }_{\gamma^{\prime}}^{M} R 2_{A}^{T 6.42}$ with turbulent viscosity for the transport of angular momentum parametrised to fit the dip in the Hyades and the Pleiades predicts rotation rates that are too slow for the lower mass models. Importantly, and for the first time, we predict that over the entire mass and metallicity range explored here, penetrative convection that is efficient at the early stages of the PMS leads to larger Li depletion in the slower rotators. This anti-correlation between the rotation rate and the Li abundance, which builds early on the PMS, remains constant over time.

\section{Model predictions for internal rotation and comparison to asteroseismic constraints}

In this section, we present the predictions of models ${ }_{v}^{M} R 1_{A}^{T 6.42}$, ${ }_{v}^{M} R 2_{A}^{T 6.42}$, and ${ }_{v^{\prime}}^{M} R 2_{A}^{T 6.42}$ with $[\mathrm{Fe} / \mathrm{H}]=+0.15 \mathrm{dex}$ (characteristic of the Hyades, Praesepe, and NGC 6819 clusters) in terms of internal rotation and tentatively compare them to the only available asteroseismic estimates of internal rotation rates in MS field stars of a similar metallicity from Benomar et al. (2015). 


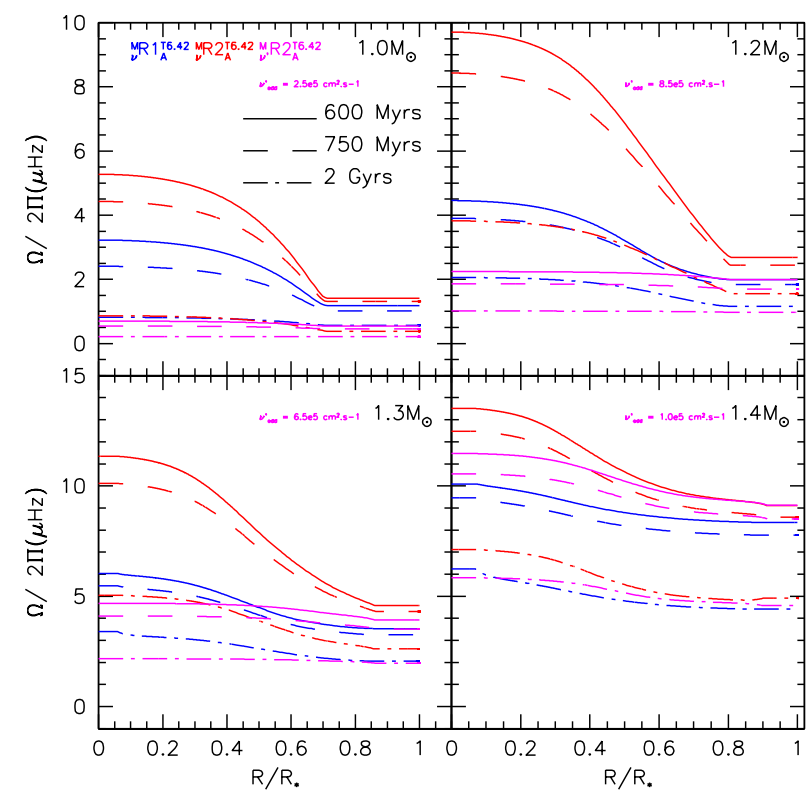

Fig. 7. Angular velocity profiles versus the radius within the star for models ${ }_{v}^{M} R 1_{A}^{T 6.42}$ (blue), ${ }_{v}^{M} R 2_{A}^{T 6.42}$ (red), and ${ }_{v^{\prime}}^{M} R 2_{A}^{T 6.42}$ (magenta) at $[\mathrm{Fe} / \mathrm{H}]=+0.15$ and for four masses: $1.0,1.2,1.3$, and $1.4 M_{\odot}$ (the value of $v_{\text {add }}^{\prime}$ used is indicated for each mass). The solid, dashed, and dot-dashed lines refer to three different ages $(0.6,0.75$, and 2 Gyrs, respectively).

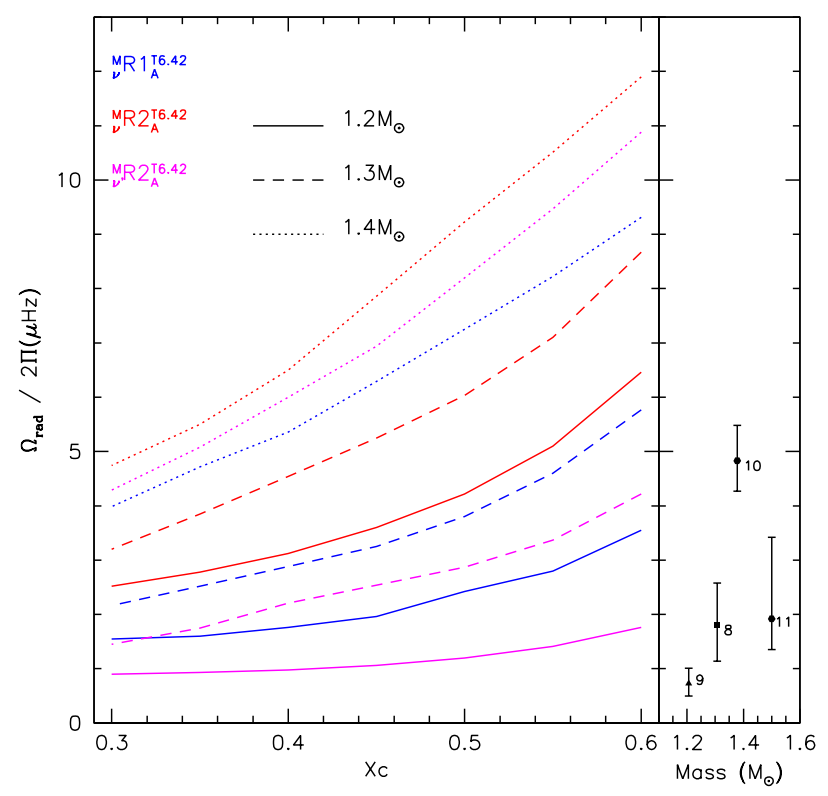

Fig. 8. Left: mean angular velocity within the stellar layers in the radiative zone as a function of the remaining central hydrogen mass fraction $X_{\mathrm{c}}$ (time increases from right to left) for the same models as in Fig. 7. Masses $1.2,1.3$, and $1.4 M_{\odot}$ are represented by full, dashed, and dotted lines respectively. Right: stars observed by Benomar et al. (2015) as indexed in the original paper (8: $M=1.307 \pm 0.027 M_{\odot}$, 9: $M=1.206 \pm 0.077 M_{\odot}, 10: M=1.378 \pm 0.028 M_{\odot}$, and $11:$ $\left.1.500 \pm 0.050 M_{\odot}\right)$.

In Fig. 7, we present the internal rotation profiles for the 1.0, 1.2, 1.3, and $1.4 M_{\odot}$ models at three different ages on the MS. For G-type stars, the ${ }_{v}^{M} R 1_{A}^{T 6.42}$ model that reproduces all surface observational constraints (see previous sections) predicts a small radial differential rotation that decreases with time and is compatible with helioseismic data as already shown in Paper I. For the more massive models corresponding to the Li-dip stars in the Hyades, Praesepe, and NGC 6819, this model predicts the same behaviour. The ${ }_{v}^{M} R 2_{A}^{T 6.42}$ model predicts larger radial differential rotation for all masses and ages than the ${ }_{v}^{M} R 1_{A}^{T 6.42}$ model, which is in agreement with the associated strong Li depletion discussed in previous sections. This results from the much higher efficiency of the vertical turbulent shear $D_{v}$ when using the Talon \& Zahn (1997) prescription (see also Paper I). For the ${ }_{v^{\prime}}^{M} R 2_{A}^{T 6.42}$ model, which introduces an enhanced parametric turbulent transport of angular momentum to fit the cold edge of the Li dip, the radial differential rotation is strongly reduced at all ages, especially in the $1.2 M_{\odot}$ model with the larger value of $v_{\text {add }}^{\prime}$, which presents an almost flat rotation profile at all ages displayed on the figure. Concerning the case of the $1.4 M_{\odot}$ model, although the angular momentum contrast between the surface and the core is of the same order in models ${ }_{v}^{M} R 1_{A}^{T 6.42}$ and ${ }_{v^{\prime}}^{M} R 2_{A}^{T 6.42}$, the angular velocity gradient near the base of the convective envelope is much larger in the later model (see change in slope in magenta profiles around $r=0.9 R_{\star}$ in Fig. 7), thus leading to an enhanced shear and associated turbulent transport in the exact region where the connection between the convective envelope and Li burning zone is made. This leads to the strong Li depletion previously discussed in this model (see Sect. 4.2). On the contrary, the shear is near to null at the base of the convective envelope in model ${ }_{v}^{M} R 1_{A}^{T 6.42}$, leading to almost no Li depletion.

Figure 8 shows the mean angular velocity in the radiative interior (i.e., excluding the convective core if present ${ }^{7}$, which is consistent with asteroseismic data that do not take it into account) as a function of the central hydrogen mass fraction in the $1.2,1.3$, and $1.4 M_{\odot}$ models at $[\mathrm{Fe} / \mathrm{H}]=+0.15 \mathrm{dex}$. We compare this to a selected sub-sample of four field stars with metallicities close to that of the Hyades for which the average rotation in the radiative interior was estimated from asteroseismology by Benomar et al. (2015). Our predictions are of the same order of magnitude as the results from Benomar et al. (2015), but the predicted rotation is faster than the values they inferred for the selected targets. Models ${ }_{\gamma^{\prime}}^{M} R 2_{A}^{T 6.42}$ lead to a better agreement with the values derived from asteroseismic data than models ${ }_{v}^{M} R 2_{A}^{T 6.42}$. A more precise comparison with the results in Benomar et al. (2015) is prevented by the use of MESA stellar models in their study including very different input physics and settings. In particular, core hydrogen mass fractions $X_{\mathrm{c}}$ cannot be compared.

Additional data for different stellar masses would be required to better discriminate between the different prescriptions for the transport of angular momentum, especially for cool stars. Nevertheless, this comparison supports the need for strong transport of angular momentum on the MS for stars in the Li-dip region, as obtained when increasing the strength of $v_{\text {add }}$ as assumed in models ${ }_{v^{\prime}}^{M} R 2_{A}^{T 6.42}$.

\section{Summary and discussion}

The need for additional transport processes beyond atomic diffusion and so-called Type-I rotation-induced processes (turbulent shear and meridional circulation) for the transport of chemicals and angular momentum has long been reported (see references in Sect. 1). This work follows Paper I analysing the impact of

7 The expression used is similar to Eq. (18) from Paper I, but starting the integration at the outer edge of the convective core when present: $\overline{\Omega_{\mathrm{rad}}}=\int_{M_{\mathrm{TCC}}}^{M_{\mathrm{BCE}}} r^{2} \Omega \mathrm{d} m / \int_{M_{\mathrm{TCC}}}^{M_{\mathrm{BCE}}} r^{2} \mathrm{~d} m$, with $M_{\mathrm{TCC}}$ being the mass coordinate of the top of the convective core and $M_{\mathrm{BCE}}$ being the mass coordinate at the base of the convective envelope. 
several processes, using state-of-the-art prescriptions for specific mechanisms (rotation-induced turbulence and penetrative convection in particular), as well as parametric prescriptions for others (parametric turbulence for chemicals and parametric viscosity for angular momentum) that were calibrated to reproduce the evolution of the surface $\mathrm{Li}$ abundance and rotation rates as well as the internal angular velocity profiles in the Sun and solar-type stars. The aim of the present paper is to study the impact of these processes (using the solar-type calibrations) on F- and G-type MS stars at different metallicities and to test them against data in Galactic open clusters over a large range in ages. Although the adopted ages come from different references and are not fully consistent, our conclusions are robust given the uncertainties on their estimate. Our predictions support, however, the Pleiades to be older than assumed in this work, which is in agreement with other age determinations for this cluster.

The so-called ${ }_{v} R 1_{A}^{T 6.42-6.5}$ models calibrated in Paper I include atomic diffusion, rotation-induced processes (meridional circulation and turbulence), penetrative convection, parametric turbulence for the transport of chemicals, and parametric viscosity for the transport of angular momentum. We have also presented ${ }_{v^{\prime}}^{M} R 2_{A}^{T 6.42}$ models which differ by the assumed prescriptions for horizontal and vertical shear-induced turbulence and were computed with different values for the parametric viscosity. We have analysed the agreement between the theoretical predictions of these two sets of models and observational data for $\mathrm{Li}, \mathrm{Be}$, and surface rotation rates available in a sample of open clusters of different ages and metallicities. We also compared the predicted internal rotation profiles with asteroseismic constraints in field MS stars with $[\mathrm{Fe} / \mathrm{H}]$ close to that of the Hyades.

Both ${ }_{v} R 1_{A}^{T 6.42-4.5}$ and ${ }_{\gamma^{\prime}}^{M} R 2_{A}^{T 6.42}$ explain the main general trends observed between $\mathrm{Li}$ depletion and stellar mass, age, rotation, and metallicity covered in this study. Thanks to the prescription we used for penetrative convection (Augustson \& Mathis 2019), an anti-correlation between Li depletion and surface rotation build up early on the PMS, and this remains throughout the evolution on the MS. Theoretical surface Li abundance and $T_{\text {eff }}$ were correlated as observed in cluster stars, with cooler and lower mass stars being more Li depleted than hotter and more massive ones (except in the Li dip when this feature is present). Our models also recover the anti-correlation between metallicity and Li depletion efficiency that is observed in open clusters stars. Metal-poor stars are less Li-depleted than their metal-rich counterparts, as expected from the metallicity dependence of the location of the base of the convective envelope that affects the PMS Li depletion. However, Li depletion in our metal-poor models is more modest than in open cluster stars.

Model ${ }_{v} R 1_{A}^{T 6.42-6.5}$ succeeds to reproduce the $\mathrm{Li}, \mathrm{Be}$, and rotation rates observations in G-type stars at all ages, and in F-type stars in the young clusters where the Li dip has not started to form yet. This is achieved thanks to the combined effects of penetrative convection, rotation-induced processes, and parametric turbulence, whose efficiencies vary with both the stellar mass and the metallicity. The impact of these mechanisms on $\mathrm{Li}$ and Be depletion becomes minute in the more massive stars that have a very thin convective envelope, more distant from the $\mathrm{Li}$ and Be burning layers. As a result, model ${ }_{v} R 1_{A}^{T 6.42-6.5}$ is not able to reproduce the $\mathrm{Li}$ and the Be dips centred around $\sim 6600 \mathrm{~K}$ that appear later on the MS, although it reproduces the rotation rates over the entire mass range covered in this study. On the other hand, model ${ }_{v}^{M} R 2_{A}^{T 6.42}$ computed with the same value of the parametric viscosity, but with different prescriptions for horizontal and vertical turbulence $\left(D_{\mathrm{h}}\right.$ and $\left.D_{\mathrm{v}}\right)$ that lead to more differential rotation (hence more efficient rotation-induced mixing) in the stellar interior, predicts too much Li depletion on the cool side of the dip, which is as expected from previous studies.

Building on the assumption that an additional transport process such as internal gravity waves would be the main driver for the transport of angular momentum in stellar interiors on the cold edge of the $\mathrm{Li}$ dip, we introduced a mass-dependent viscosity $v_{\text {add }}^{\prime}$ with a maximum efficiency at $\approx 5900 \mathrm{~K}$ as predicted in Talon \& Charbonnel (2003) and Talon \& Charbonnel (2004) for the IGW excitation by the convective envelope and their luminosity. Although the assumption we make of a parametric viscosity $v_{\text {add }}^{\left({ }^{\prime}\right)}$ being uniform within the radiative interior and constant with time is a very crude parametrisation of a transport of angular momentum within stars, the ${ }_{v^{\prime}}^{M} R 2_{A}^{T 6.42}$ model predicts a good agreement with $\mathrm{Li}$ abundances on the cold edge of the dip in open clusters of different ages at the metallicity of the Hyades (this was not tested for other metallicities). This is due to the flattening of the angular velocity gradient within the stellar interior, and in particular between the base of the convective envelope and the Li burning depth, implied by higher values of the parametric viscosity. A further improvement to our model now requires the full treatment of the transport of angular momentum by IGWs as in Charbonnel \& Talon (2005), also taking into account recent developments on the generation and the behaviour of IGWs (e.g., Pinçon et al. 2016; Augustson et al. 2020; Ratnasingam et al. 2020). The Tayler-Spruit dynamo process that was updated in recent studies (e.g., Fuller et al. 2019; Eggenberger et al. 2019a,b,c) is also a candidate for additional transport of angular momentum and should also be tested regarding the results of the present work. The Be depletion obtained with model ${ }_{v^{\prime}}^{M} R 2_{A}^{T 6.42}$ is on the other hand too modest to explain the depth of the Be dip observed in a couple of clusters. The acquisition of more Be observational data in open clusters of different ages and metallicities, in addition to those in field stars (e.g., Delgado Mena et al. 2012), would be an important key to better constrain models.

Importantly, the transport of angular momentum simulated with the parametric viscosity values adopted in our different models is much more efficient than that driven by meridional circulation and turbulence. Both the ${ }_{v} R 1_{A}^{T 6.42}$ and the ${ }_{v^{\prime}}^{M} R 2_{A}^{T 6.42}$ models predict mean angular rotation velocity in the radiative interior that are compatible with the values inferred from asteroseismology in field MS with the same metallicity as the Hyades and the Pleiades. Additional asteroseismic data to probe the internal rotation of young MS stars over a large metallicity range would be valuable.

Reflecting on the modelling of turbulent shear, we have to conclude that none of the combinations for the prescriptions of the horizontal and vertical turbulence $\left(D_{\mathrm{h}}\right.$ and $\left.D_{v}\right)$ we used is able to reconcile all the surface constraints used in this study. Model ${ }_{v} R 1_{A}^{T 6.42-6.5}$, tailored for solar-type stars, includes the Mathis et al. (2018) prescription for the horizontal shear diffusivity $D_{\mathrm{h}}$, which is the only prescription including the contributions of both the horizontal and vertical shear on the horizontal turbulent transport, and the Zahn (1992) prescription for the vertical shear diffusivity $D_{\mathrm{v}}$, which does not take into account the effects of thermal and molecular diffusivity on the vertical turbulent transport, but it is the only one that has been validated by direct numerical simulations (Prat \& Lignières 2013; Garaud et al. 2017). This model thus includes the apparently most robust available prescriptions, but it cannot explain the Li dip even when combined with additional sources for turbulent transport such as $D_{\mathrm{T}_{0}}$ and $v_{\text {add }}$. 
On the other hand, the use of the Zahn (1992) prescription for the horizontal shear diffusivity $\left(D_{\mathrm{h}}\right)$ and that of Talon \& Zahn (1997), which includes the effect of thermal and molecular diffusion for the vertical shear diffusivity $\left(D_{\mathrm{v}}\right)$ in model ${ }_{v^{\prime}}^{M} R 2_{A}^{T 6.42}$, requires higher values for the parametric viscosity for the internal transport of angular momentum, with a mass-dependent efficiency as expected from IGW. The choice of these prescriptions thus impacts our ability to constrain the other transport processes (see Meynet et al. 2013; Amard et al. 2016) as additionally illustrated by the predictions obtained in this work and for instance by Semenova et al. (2020) for the specific case of NGC 2420. Insight and validation of the different available prescriptions for the vertical turbulent shear transport from hydrodynamicists and multi-dimensional numerical simulations, similar to the ongoing work on the horizontal turbulent shear (Park et al. 2020, 2021; Prat \& Mathis 2021), are now mandatory in order to overcome this impasse.

Finally, two additional promising leads to understand the formation of the $\mathrm{Li}$ and $\mathrm{Be}$ dips, in particular, should be explored, which were neglected here. First, we do not consider radiative accelerations on heavy elements. Their effects are, however, nonnegligible for stars with an effective temperature higher than $\approx 6800 \mathrm{~K}$, which corresponds to $\approx 1.4 M_{\odot}$ at solar metallicity, close to the hot edge of the Li dip (e.g., Richer \& Michaud 1993; Deal et al. 2018, 2020). Their impact on the stellar opacity could partly modify some of our conclusions concerning the most massive models. Second, the process sustaining the parametric turbulence used in this work and others in the literature has yet to be identified. In particular, even if the tachocline mixing (Spiegel \& Zahn 1992; Brun et al. 1999; Garaud 2020) was shown to not be adapted in solar-type stars (Paper I), this process could play a role in the building of the $\mathrm{Li}$ and $\mathrm{Be}$ dips because of its dependence at the latitudinal differential rotation that is predicted to scale inversely with rotation for F-type stars (Augustson et al. 2012) compared to G-type stars.

Acknowledgements. We thank J.W. Ferguson for providing us with lowtemperature opacity tables adapted to the solar mixture we adopt in this work E. Semenova for providing us observational data for NGC 2420 open cluster, J.D. Cummings and collaborators for providing the $\log g$ values for their Hyades and Praesepe sample, L. Amard and P. Eggenberger for fruitful discussions, and the anonymous referee for constructive comments on the manuscript. This work was supported by the Swiss National Science Foundation (Project 200020192039 PI C.C.), by CNES (funding of AP at LUPM) focussed on the PLATO mission, and by the Agence Nationale de la Recherche grant POPSYCLE number ANR-19-CE31-0022. This research has made use of NASA's Astrophysics Data System Bibliographic Services.

\section{References}

Aerts, C., Mathis, S., \& Rogers, T. M. 2019, ARA\&A, 57, 35

Agüeros, M. A., Covey, K. R., Lemonias, J. J., et al. 2011, ApJ, 740, 110

Allain, S. 1998, A\&A, 333, 629

Amard, L., Palacios, A., Charbonnel, C., Gallet, F., \& Bouvier, J. 2016, A\&A, 587, A105

Amard, L., Palacios, A., Charbonnel, C., et al. 2019, A\&A, 631, A77

Anthony-Twarog, B. J., Deliyannis, C. P., Twarog, B. A., Croxall, K. V., \& Cummings, J. D. 2009, AJ, 138, 1171

Anthony-Twarog, B. J., Deliyannis, C. P., Harmer, D., et al. 2018, AJ, 156, 37

Arancibia-Silva, J., Bouvier, J., Bayo, A., et al. 2020, A\&A, 635, L13

Asplund, M., Grevesse, N., Sauval, A. J., \& Scott, P. 2009, ARA\&A, 47, 481

Augustson, K. C., \& Mathis, S. 2019, ApJ, 874, 83

Augustson, K. C., Brown, B. P., Brun, A. S., Miesch, M. S., \& Toomre, J. 2012, ApJ, 756, 169

Augustson, K. C., Mathis, S., \& Astoul, A. 2020, ApJ, 903, 90

Balachandran, S. 1995, ApJ, 446, 203

Balachandran, S. C., \& Bell, R. A. 1998, Nature, 392, 791

Balachandran, S., Lambert, D. L., \& Stauffer, J. R. 1988, ApJ, 333, 267
Balachandran, S. C., Mallik, S. V., \& Lambert, D. L. 2011, MNRAS, 410, 2526 Baraffe, I., Pratt, J., Goffrey, T., et al. 2017, ApJ, 845, L6

Barrado y Navascués, D., Deliyannis, C. P., \& Stauffer, J. R. 2001, ApJ, 549, 452 Barrado y Navascués, D., Stauffer, J. R., \& Jayawardhana, R. 2004, ApJ, 614, 386

Benomar, O., Takata, M., Shibahashi, H., Ceillier, T., \& García, R. A. 2015, MNRAS, 452, 2654

Bodenheimer, P. 1965, ApJ, 142, 451

Boesgaard, A. M. 1976, PASP, 88, 353

Boesgaard, A. M. 1987, PASP, 99, 1067

Boesgaard, A. M. 1991, ApJ, 370, L95

Boesgaard, A. M., \& Tripicco, M. J. 1986, ApJ, 302, L49

Boesgaard, A. M., Deliyannis, C. P., King, J. R., \& Stephens, A. 2001, ApJ, 553, 754

Boesgaard, A. M., Armengaud, E., \& King, J. R. 2003a, ApJ, 583, 955

Boesgaard, A. M., Armengaud, E., \& King, J. R. 2003b, ApJ, 582, 410

Boesgaard, A. M., Armengaud, E., King, J. R., Deliyannis, C. P., \& Stephens, A. 2004a, ApJ, 613, 1202

Boesgaard, A. M., Armengaud, E., \& King, J. R. 2004b, ApJ, 605, 864 Boesgaard, A. M., Lum, M. G., Deliyannis, C. P., et al. 2016, ApJ, 830, 49 Boesgaard, A. M., Lum, M. G., \& Deliyannis, C. P. 2020, ApJ, 888, 28 Bossini, D., Vallenari, A., Bragaglia, A., et al. 2019, A\&A, 623, A108 Bouvier, J. 2008, A\&A, 489, L53

Bouvier, J., Barrado, D., Moraux, E., et al. 2018, A\&A, 613, A63

Brun, A. S., Turck-Chièze, S., \& Zahn, J. P. 1999, ApJ, 525, 1032

Brun, A. S., Strugarek, A., Varela, J., et al. 2017, ApJ, 836, 192

Cantat-Gaudin, T., Anders, F., Castro-Ginard, A., et al. 2020, A\&A, 640, A1

Carlsson, M., Rutten, R. J., Bruls, J. H. M. J., \& Shchukina, N. G. 1994, A\&A, 288,860

Casagrande, L., Ramírez, I., Meléndez, J., Bessell, M., \& Asplund, M. 2010, A\&A, 512, A54

Castro, M., Vauclair, S., Richard, O., \& Santos, N. C. 2009, A\&A, 494, 663

Castro, M., Duarte, T., Pace, G., \& do Nascimento, J. D. 2016, A\&A, 590, A94

Cayrel, R., Cayrel de Strobel, G., Campbell, B., \& Dappen, W. 1984, ApJ, 283, 205

Charbonneau, P., \& MacGregor, K. B. 1993, ApJ, 417, 762

Charbonneau, P., \& Michaud, G. 1990, ApJ, 352, 681

Charbonnel, C., \& Talon, S. 2005, Science, 309, 2189

Charbonnel, C., Vauclair, S., \& Zahn, J. P. 1992, A\&A, 255, 191

Cummings, J. D., Deliyannis, C. P., Anthony-Twarog, B., Twarog, B., \& Maderak, R. M. 2012, AJ, 144, 137

Cummings, J. D., Deliyannis, C. P., Maderak, R. M., \& Steinhauer, A. 2017, AJ, 153,128

Deal, M., Alecian, G., Lebreton, Y., et al. 2018, A\&A, 618, A10

Deal, M., Goupil, M. J., Marques, J. P., Reese, D. R., \& Lebreton, Y. 2020, A\&A, 633, A23

Decressin, T., Mathis, S., Palacios, A., et al. 2009, A\&A, 495, 271

Delgado Mena, E., Israelian, G., González Hernández, J. I., Santos, N. C., \& Rebolo, R. 2012, ApJ, 746, 47

Deliyannis, C. P., Boesgaard, A. M., Stephens, A., et al. 1998, ApJ, 498, L147

Deliyannis, C. P., Pinsonneault, M. H., \& Charbonnel, C. 2000, in The Light Elements and their Evolution, eds. L. da Silva, R. de Medeiros, \& M. Spite, IAU Symp., 198, 61

Deliyannis, C. P., Anthony-Twarog, B. J., Lee-Brown, D. B., \& Twarog, B. A. 2019, AJ, 158, 163

Delorme, P., Collier Cameron, A., Hebb, L., et al. 2011, MNRAS, 413, 2218

Douglas, S. T., Agüeros, M. A., Covey, K. R., et al. 2016, ApJ, 822, 47

Douglas, S. T., Agüeros, M. A., Covey, K. R., \& Kraus, A. 2017, ApJ, 842, 83

Douglas, S. T., Curtis, J. L., Agüeros, M. A., et al. 2019, ApJ, 879, 100

Dumont, T., Palacios, A., Charbonnel, C., et al. 2021, A\&A, 646, A48

Duncan, D. K., \& Jones, B. F. 1983, ApJ, 271, 663

Eff-Darwich, A., Korzennik, S. G., Jiménez-Reyes, S. J., \& García, R. A. 2008, ApJ, 679, 1636

Eggenberger, P., Maeder, A., \& Meynet, G. 2005, A\&A, 440, L9

Eggenberger, P., Meynet, G., Maeder, A., et al. 2010, A\&A, 519, A116

Eggenberger, P., Haemmerlé, L., Meynet, G., \& Maeder, A. 2012a, A\&A, 539, A70

Eggenberger, P., Montalbán, J., \& Miglio, A. 2012b, A\&A, 544, L4

Eggenberger, P., Buldgen, G., \& Salmon, S. J. A. J. 2019a, A\&A, 626, L1

Eggenberger, P., Deheuvels, S., Miglio, A., et al. 2019b, A\&A, 621, A66

Eggenberger, P., den Hartogh, J. W., Buldgen, G., et al. 2019c, A\&A, 631, L6

Elsworth, Y., Howe, R., Isaak, G. R., et al. 1995, Nature, 376, 669

François, P., Pasquini, L., Biazzo, K., Bonifacio, P., \& Palsa, R. 2013, A\&A, 552, A136

Fuller, J., Piro, A. L., \& Jermyn, A. S. 2019, MNRAS, 485, 3661

Gallet, F., \& Bouvier, J. 2015, A\&A, 577, A98

Garaud, P. 2020, Astrophys. Space Sci. Proc., 57, 207

Garaud, P., Gagnier, D., \& Verhoeven, J. 2017, ApJ, 837, 133 
García, R. A., \& Ballot, J. 2019, Liv. Rev. Sol. Phys., 16, 4

Garcia Lopez, R. J., Rebolo, R., \& Martin, E. L. 1994, A\&A, 282, 518

Godoy-Rivera, D., Pinsonneault, M. H., \& Rebull, L. M. 2021, ApJS, submitted [arXiv:2101.01183]

Gutiérrez Albarrán, M. L., Montes, D., Gómez Garrido, M., et al. 2020, A\&A, 643, A71

Guzik, J. A., \& Mussack, K. 2010, ApJ, 713, 1108

Hartman, J. D., Gaudi, B. S., Holman, M. J., et al. 2008, ApJ, 675, 1254

Hartman, J. D., Bakos, G. Á., Kovács, G., \& Noyes, R. W. 2010, MNRAS, 408, 475

Hobbs, L. M., \& Pilachowski, C. 1986, ApJ, 309, L17

Huang, Y., Liu, X. W., Yuan, H. B., et al. 2015, MNRAS, 454, 2863

Irwin, J., \& Bouvier, J. 2009, in The Ages of Stars, eds. E. E. Mamajek, D. R. Soderblom, \& R. F. G. Wyse, 258, 363

Irwin, J., Hodgkin, S., Aigrain, S., et al. 2008, MNRAS, 384, 675

Irwin, J., Aigrain, S., Bouvier, J., et al. 2009, MNRAS, 392, 1456

Jackson, R. J., Jeffries, R. D., Deliyannis, C. P., Sun, Q., \& Douglas, S. T. 2019 , MNRAS, 483, 1125

Jaehnig, K., Somers, G., \& Stassun, K. G. 2019, ApJ, 879, 39

Jeffries, R. D. 1997, MNRAS, 292, 177

Jeffries, R. D., Totten, E. J., Harmer, S., \& Deliyannis, C. P. 2002, MNRAS, 336, 1109

Jeffries, R. D., Jackson, R. J., Sun, Q., \& Deliyannis, C. P. 2021, MNRAS, 500, 1158

Jones, B. F., Fischer, D., \& Soderblom, D. R. 1999, AJ, 117, 330

Jørgensen, A. C. S., \& Weiss, A. 2018, MNRAS, 481, 4389

Kawaler, S. D. 1988, ApJ, 333, 236

Kosovichev, A. G. 1988, Sov. Astron. Lett., 14, 145

Kraft, R. P. 1967, ApJ, 150, 551

Krishnamurthi, A., Pinsonneault, M. H., Barnes, S., \& Sofia, S. 1997, ApJ, 480, 303

Lagarde, N., Decressin, T., Charbonnel, C., et al. 2012, A\&A, 543, A108

Maeder, A., \& Zahn, J.-P. 1998, A\&A, 334, 1000

Marques, J. P., Goupil, M. J., Lebreton, Y., et al. 2013, A\&A, 549, A74

Mathis, S. 2013, in Transport Processes in Stellar Interiors, eds. M. Goupil, K. Belkacem, C. Neiner, F. Lignières, \& J. J. Green, 865, 23

Mathis, S., \& Zahn, J. P. 2004, A\&A, 425, 229

Mathis, S., Prat, V., Amard, L., et al. 2018, A\&A, 620, A22

Mathur, S., Eff-Darwich, A., García, R. A., \& Turck-Chièze, S. 2008, A\&A, 484, 517

Matt, S. P., Brun, A. S., Baraffe, I., Bouvier, J., \& Chabrier, G. 2015, ApJ, 799, L23

Matteucci, F., D’Antona, F., \& Timmes, F. X. 1995, A\&A, 303, 460

Meibom, S., Mathieu, R. D., \& Stassun, K. G. 2009, ApJ, 695, 679

Meibom, S., Barnes, S. A., Latham, D. W., et al. 2011, ApJ, 733, L9

Meibom, S., Barnes, S. A., Platais, I., et al. 2015, Am. Astron. Soc. Meeting Abstr., 225, 449.09

Meléndez, J., Schuster, W. J., Silva, J. S., et al. 2010, A\&A, 522, A98

Meynet, G., Ekstrom, S., Maeder, A., et al. 2013, in Models of Rotating Massive

Stars: Impacts of Various Prescriptions, eds. M. Goupil, K. Belkacem,

C. Neiner, F. Lignières, \& J. J. Green, 865, 3

Michaud, G. 1986, ApJ, 302, 650

Montalban, J. 1994, A\&A, 281, 421

Montalbán, J., \& Rebolo, R. 2002, A\&A, 386, 1039

Montalban, J., \& Schatzman, E. 1996, A\&A, 305, 513

Montalbán, J., \& Schatzman, E. 2000, A\&A, 354, 943

Netopil, M., Paunzen, E., Heiter, U., \& Soubiran, C. 2016, A\&A, 585, A150

Palacios, A., Talon, S., Charbonnel, C., \& Forestini, M. 2003, A\&A, 399, 603

Palacios, A., Charbonnel, C., Talon, S., \& Siess, L. 2006, A\&A, 453, 261

Pace, G., Castro, M., Meléndez, J., Théado, S., \& do Nascimento, Jr., J. D. 2012, A\&A, 541, A150

Paquette, C., Pelletier, C., Fontaine, G., \& Michaud, G. 1986, ApJS, 61, 177

Park, J., Prat, V., \& Mathis, S. 2020, A\&A, 635, A133

Park, J., Prat, V., Mathis, S., \& Bugnet, L. 2021, A\&A, 646, A64
Pinçon, C., Belkacem, K., \& Goupil, M. J. 2016, A\&A, 588, A122 Pinsonneault, M. 1997, ARA\&A, 35, 557

Pinsonneault, M. H., Kawaler, S. D., \& Demarque, P. 1990, ApJS, 74, 501 Prantzos, N. 2012, A\&A, 542, A67

Prat, V., \& Lignières, F. 2013, A\&A, 551, L3

Prat, V., \& Mathis, S. 2021, A\&A, 649, A62

Pratt, J., Baraffe, I., Goffrey, T., et al. 2017, A\&A, 604, A125

Randich, S., Pallavicini, R., Meola, G., Stauffer, J. R., \& Balachandran, S. C. 2001, A\&A, 372, 862

Randich, S., Pasquini, L., Franciosini, E., et al. 2020, A\&A, 640, L1

Ratnasingam, R. P., Edelmann, P. V. F., \& Rogers, T. M. 2020, MNRAS, 497, 4231

Richard, O., Vauclair, S., Charbonnel, C., \& Dziembowski, W. A. 1996, A\&A 312, 1000

Richard, O., Michaud, G., Richer, J., et al. 2002, ApJ, 568, 979

Richard, O., Michaud, G., \& Richer, J. 2005, ApJ, 619, 538

Richer, J., \& Michaud, G. 1993, ApJ, 416, 312

Richer, J., Michaud, G., Rogers, F., et al. 1998, ApJ, 492, 833

Richer, J., Michaud, G., \& Turcotte, S. 2000, ApJ, 529, 338

Romano, D., Matteucci, F., Ventura, P., \& D'Antona, F. 2001, A\&A, 374, 646

Ruediger, G., \& Kitchatinov, L. L. 1996, ApJ, 466, 1078

Schatzman, E. 1959, The Hertzsprung-Russell Diagram, ed. J. L. Greenstein, 10, 129

Schatzman, E. 1962, Ann. Astrophys., 25, 18

Schlattl, H., \& Weiss, A. 1999, A\&A, 347, 272

Semenova, E., Bergemann, M., Deal, M., et al. 2020, A\&A, 643, A164

Sestito, P., \& Randich, S. 2005, A\&A, 442, 615

Siess, L., Dufour, E., \& Forestini, M. 2000, A\&A, 358, 593

Skumanich, A. 1972, ApJ, 171, 565

Soderblom, D. R., Oey, M. S., Johnson, D. R. H., \& Stone, R. P. S. 1990, AJ, 99, 595

Soderblom, D. R., Pilachowski, C. A., Fedele, S. B., \& Jones, B. F. 1993a, AJ, 105, 2299

Soderblom, D. R., Fedele, S. B., Jones, B. F., Stauffer, J. R., \& Prosser, C. F. 1993b, AJ, 106, 1080

Somers, G., \& Pinsonneault, M. H. 2015, MNRAS, 449, 4131

Somers, G., \& Pinsonneault, M. H. 2016, ApJ, 829, 32

Somers, G., \& Stassun, K. G. 2017, AJ, 153, 101

Spada, F., Gellert, M., Arlt, R., \& Deheuvels, S. 2016, A\&A, 589, A23

Spiegel, E. A., \& Zahn, J. P. 1992, A\&A, 265, 106

Spite, F., \& Spite, M. 1982, A\&A, 115, 357

Spruit, H. C. 2002, A\&A, 381, 923

Steinhauer, A., \& Deliyannis, C. P. 2004, ApJ, 614, L65

Swenson, F. J., Faulkner, J., Rogers, F. J., \& Iglesias, C. A. 1994, ApJ, 425, 286

Talon, S., \& Charbonnel, C. 1998, in Cool Stars, Stellar Systems, and the Sun, eds. R. A. Donahue, \& J. A. Bookbinder, ASP Conf. Ser., 154, 973

Talon, S., \& Charbonnel, C. 2003, A\&A, 405, 1025

Talon, S., \& Charbonnel, C. 2004, A\&A, 418, 1051

Talon, S., \& Charbonnel, C. 2005, A\&A, 440, 981

Talon, S., \& Charbonnel, C. 2010, in Light Elements in the Universe, eds. C. Charbonnel, M. Tosi, F. Primas, \& C. Chiappini, IAU Symp., 268, 365

Talon, S., \& Zahn, J. P. 1997, A\&A, 317, 749

Thorburn, J. A., Hobbs, L. M., Deliyannis, C. P., \& Pinsonneault, M. H. 1993, ApJ, 415, 150

Thoul, A. A., Bahcall, J. N., \& Loeb, A. 1994, ApJ, 421, 828

Travaglio, C., Randich, S., Galli, D., et al. 2001, ApJ, 559, 909

Turcotte, S., Richer, J., \& Michaud, G. 1998, ApJ, 504, 559

Ujjwal, K., Kartha, S. S., Mathew, B., Manoj, P., \& Narang, M. 2020, AJ, 159, 166

Wallerstein, G., Herbig, G. H., \& Conti, P. S. 1965, ApJ, 141, 610

Wang, E. X., Nordlander, T., Asplund, M., et al. 2021, MNRAS, 500, 2159

Weber, E. J., \& Davis, Jr., L. 1967, ApJ, 148, 217

Zahn, J. P. 1992, A\&A, 265, 115

Zhang, Q.-S., Li, Y., \& Christensen-Dalsgaard, J. 2019, ApJ, 881, 103 


\section{Appendix A: Model predictions}

Table A.1. ${ }_{v}^{M} R 1_{A}^{T 6.42}$ model predictions for the different clusters with lithium abundances presented in Table 1 and in Figs. 5 and 6.

\begin{tabular}{|c|c|c|c|c|}
\hline Cluster & $\operatorname{Mass}\left(M_{\odot}\right)$ & $T_{\text {eff }}(\mathrm{K})$ & $\mathrm{A}(\mathrm{Li})$ & $\mathrm{P}_{\mathrm{rot}}(\mathrm{d})$ \\
\hline \multirow{6}{*}{ IC 2602 / IC 2391} & 0.8 & 4347 & 1.54 & 1.6 \\
\hline & 0.9 & 5099 & 2.47 & 1.5 \\
\hline & 1.0 & 5742 & 2.82 & 1.2 \\
\hline & 1.1 & 5937 & 3.06 & 1.2 \\
\hline & 1.2 & 6247 & 3.15 & 1.3 \\
\hline & 1.3 & 6564 & 3.19 & 1.1 \\
\hline \multirow{6}{*}{ Pleaides } & 0.8 & 4855 & 1.50 & 1.3 \\
\hline & 0.9 & 5275 & 2.46 & 1.5 \\
\hline & 1.0 & 5649 & 2.81 & 1.8 \\
\hline & 1.1 & 5982 & 3.06 & 2.4 \\
\hline & 1.2 & 6284 & 3.14 & 1.9 \\
\hline & 1.3 & 6578 & 3.19 & 0.9 \\
\hline \multirow{7}{*}{$\alpha$ Per } & 0.8 & 4685 & -0.49 & 1.3 \\
\hline & 0.9 & 5103 & 1.51 & 1.5 \\
\hline & 1.0 & 5470 & 2.28 & 1.8 \\
\hline & 1.1 & 5806 & 2.79 & 2.4 \\
\hline & 1.2 & 6104 & 2.95 & 1.9 \\
\hline & 1.3 & 6388 & 3.09 & 0.9 \\
\hline & 1.4 & 6670 & 3.19 & 2.3 \\
\hline \multirow{5}{*}{ M 35} & 0.8 & 5072 & 2.67 & 2.3 \\
\hline & 0.9 & 5512 & 3.00 & 2.6 \\
\hline & 1.0 & 5894 & 3.13 & 3.4 \\
\hline & 1.1 & 6225 & 3.22 & 3.5 \\
\hline & 1.2 & 6538 & 3.25 & 2.4 \\
\hline \multirow{5}{*}{ Coma Ber } & 0.9 & 5284 & 2.13 & 10.5 \\
\hline & 1.0 & 5671 & 2.66 & 8.5 \\
\hline & 1.1 & 6001 & 2.92 & 6.6 \\
\hline & 1.2 & 6305 & 3.01 & 4.3 \\
\hline & 1.3 & 6597 & 3.10 & 1.1 \\
\hline \multirow{6}{*}{ UMa } & 0.8 & 4953 & 1.64 & 11.8 \\
\hline & 0.9 & 5402 & 2.62 & 9.3 \\
\hline & 1.0 & 5789 & 2.84 & 8.0 \\
\hline & 1.1 & 6121 & 3.03 & 5.7 \\
\hline & 1.2 & 6429 & 3.08 & 3.2 \\
\hline & 1.3 & 6733 & 3.14 & 1.3 \\
\hline \multirow{8}{*}{ Hyades } & 0.8 & 4665 & -2.11 & 12.4 \\
\hline & 0.9 & 5108 & 0.85 & 10.5 \\
\hline & 1.0 & 5497 & 1.99 & 8.5 \\
\hline & 1.1 & 5829 & 2.65 & 6.6 \\
\hline & 1.2 & 6127 & 2.83 & 4.3 \\
\hline & 1.3 & 6407 & 2.96 & 3.3 \\
\hline & 1.4 & 6679 & 3.07 & 1.4 \\
\hline & 1.5 & 6964 & 3.08 & 0.8 \\
\hline \multirow{6}{*}{ NGC 6633} & 0.8 & 4955 & 1.38 & 14.3 \\
\hline & 0.9 & 5406 & 2.55 & 10.5 \\
\hline & 1.0 & 5793 & 2.79 & 9.0 \\
\hline & 1.1 & 6126 & 2.98 & 6.3 \\
\hline & 1.2 & 6434 & 3.03 & 3.5 \\
\hline & 1.3 & 6737 & 3.09 & 1.4 \\
\hline \multirow{8}{*}{ Praesepe } & 0.8 & 4667 & -2.73 & 15.2 \\
\hline & 0.9 & 5112 & 0.57 & 12.1 \\
\hline & 1.0 & 5501 & 1.91 & 9.7 \\
\hline & 1.1 & 5833 & 2.60 & 7.2 \\
\hline & 1.2 & 6132 & 2.78 & 4.7 \\
\hline & 1.3 & 6410 & 2.91 & 3.6 \\
\hline & 1.4 & 6677 & 3.03 & 0.9 \\
\hline & 1.5 & 6944 & 3.03 & 0.8 \\
\hline \multirow{4}{*}{ NGC 6819} & 0.9 & 5198 & -1.32 & 24.3 \\
\hline & 1.0 & 5594 & 1.74 & 19.1 \\
\hline & 1.1 & 5925 & 2.29 & 13.1 \\
\hline & 1.2 & 6218 & 2.47 & 8.6 \\
\hline
\end{tabular}


T. Dumont et al.: Lithium depletion and angular momentum transport in F-type and G-type stars in Galactic open clusters

Table A.1. continued.

\begin{tabular}{ccccc}
\hline \hline Cluster & Mass $\left(M_{\odot}\right)$ & $T_{\text {eff }}(\mathrm{K})$ & $\mathrm{A}(\mathrm{Li})$ & $\mathrm{P}_{\text {rot }}(\mathrm{d})$ \\
\hline NGC 6819 & 1.3 & 6457 & 2.57 & 4.5 \\
& 1.4 & 6634 & 2.70 & 1.8 \\
\hline \multirow{4}{*}{ NGC 2420 } & 0.9 & 5391 & 1.10 & 29.1 \\
& 1.0 & 5785 & 2.17 & 18.9 \\
& 1.1 & 6118 & 2.45 & 11.9 \\
& 1.2 & 6398 & 2.46 & 6.9 \\
M67 & 1.3 & 6598 & 2.54 & 3.0 \\
& 1.4 & 6741 & 2.58 & 1.2 \\
\hline & 0.9 & 5369 & -0.24 & 49.5 \\
& 1.0 & 5765 & 1.78 & 28.7 \\
NGC 2243 & 1.1 & 6083 & 2.05 & 17.5 \\
& 1.2 & 6240 & 2.10 & 11.7 \\
\hline & 0.8 & 5293 & 0.71 & 48.5 \\
& 0.9 & 5766 & 1.74 & 26.6 \\
& 1.0 & 6152 & 2.06 & 15.3 \\
& 1.1 & 6455 & 2.04 & 7.2 \\
\hline
\end{tabular}

